\title{
Holography of massive M2-brane theory: non-linear extension
}

\author{
O-Kab Kwon ${ }^{1, \mathrm{a}}$, Dongmin Jang ${ }^{1, \mathrm{~b}}$, Yoonbai Kim ${ }^{1, \mathrm{c}}$, D. D. Tolla ${ }^{1,2, \mathrm{~d}}$ \\ ${ }^{1}$ Department of Physics, BK21 Physics Research Division, Institute of Basic Science, Sungkyunkwan University, Suwon 440-746, South Korea \\ ${ }^{2}$ University College, Sungkyunkwan University, Suwon 440-746, South Korea
}

Received: 29 May 2018 / Accepted: 11 October 2018 / Published online: 25 October 2018

(c) The Author(s) 2018

\begin{abstract}
We investigate the gauge/gravity duality between the $\mathcal{N}=6$ mass-deformed ABJM theory with $\mathrm{U}_{k}(N) \times$ $\mathrm{U}_{-k}(N)$ gauge symmetry and the 11-dimensional supergravity on LLM geometries with $\mathrm{SO}(2,1) \times \mathrm{SO}(4) / \mathbb{Z}_{k} \times \mathrm{SO}(4) / \mathbb{Z}_{k}$ isometry, in terms of a KK holography, which involves quadratic order field redefinitions. We establish the quadratic order KK mappings for various gauge invariant fields in order to obtain the canonical 4-dimensional gravity equations of motion and to reduce the LLM solutions to an asymptotically $\mathrm{AdS}_{4}$ gravity solutions. The non-linearity of the KK maps indicates that we can observe the true purpose of the non-linear KK holography of the LLM solutions. We read the vacuum expectation value of conformal dimension two operator from the asymptotically $\mathrm{AdS}_{4}$ gravity solutions. For the LLM solutions which are represented by squareshaped Young diagrams, we compare the vacuum expectation value obtained from the holographic procedure with the result obtained from the field theory, which is given by $\left\langle\mathcal{O}^{(\Delta=2)}\right\rangle=\sqrt{k} N^{\frac{3}{2}} f_{(\Delta=2)}+\mathcal{O}(N)$, where $f_{\Delta}$ is independent of $N$. Based on this result, we examine the gauge/gravity duality in the large $N$ limit and finite $k$. We also show that the vacuum expectation values of the massive KK graviton modes are vanishing as expected by the supersymmetry.
\end{abstract}

\section{Contents}

1 Introduction . . . . . . . . . . . . 1

2 KK reduction of 11-dimensional gravity . . . . . . 2

2.1 Field equations at quadratic order . . . . . . . . 2

2.2 Quadratic order equations for KK modes . . . . 4

2.2.1 Spin-zero field equations . . . . . . . . 5

2.2.2 Spin-two field equations . . . . . . . 6

\footnotetext{
a e-mail: okab@skku.edu

be-mail: dongmin@skku.edu

ce-mail: yoonbai@skku.edu

de-mail: ddtolla@skku.edu
}

3 Gauge invariant operators and Vevs in mABJM theory 8 3.1 Vacua in the mABJM theory . . . . . . . . 8

3.2 Gauge invariant operators in the ABJM theory . 9

4 Asymptotic behavior of LLM geometries and 4dimensional KK modes . . . . . . . . . . 10

5 Vevs of CPOs and GKP-W relation . . . . . . . 11

6 Conclusion . . . . . . . . . . . . . . 13



A $C^{I_{1}=4}$ and $C^{(\Delta=2)} \ldots \ldots \ldots \ldots \ldots \ldots$

References . . . . . . . . . . . . . 15

\section{Introduction}

AdS/CFT correspondence [1-3] and its various deformations have been a central paradigm for the past two decades in theoretical physics. Among the deformations, we consider the supersymmetry preserving mass deformation $[4,5]$ of the 3dimensional $\mathcal{N}=6 \mathrm{U}_{k}(N) \times \mathrm{U}_{-k}(N)$ Aharony-BergmanJafferis-Maldacena (ABJM) theory with Chern-Simons level $k$ [6], which is dual to the 11-dimensional supergravity on the Lin-Lunin-Maldacena (LLM) geometries [7] with $\mathbb{Z}_{k}$ orbifold and $\mathrm{SO}(2,1) \times \mathrm{SO}(4) / \mathbb{Z}_{k} \times \mathrm{SO}(4) / \mathbb{Z}_{k}$ isometry. The correspondence between the supersymmetric vacua of the massdeformed ABJM theory (mABJM) and the LLM geometries with $\mathbb{Z}_{k}$ orbifold was reported in [8].

Recently, we have disclosed more evidence for the gauge/gravity duality between the mABJM theory and the 11-dimensional supergravity on the LLM geometry with $\mathrm{SO}(2,1) \times \mathrm{SO}(4) / \mathbb{Z}_{k} \times \mathrm{SO}(4) / \mathbb{Z}_{k}$ isometry $[9,10]$. We calculated the vacuum expectation values (vevs) of a chiral primary operator (CPO) of conformal dimension $\Delta=1$, from all supersymmetric vacua of the mABJM theory in the large $N$ limit and from the LLM solutions in the 11dimensional supergravity in terms of the gauge/gravity dictionary $[2,3]$. In order to show the duality, we defined the 4-dimensional dual scalar modes obtained from the procedure of the Kaluza-Klein (KK) holography [11-13] for the 11-dimensional supergravity. We found an exact dual rela- 
tion between the two results for all possible supersymmetric solutions in both sides in the large $N$ limit.

In the case of the CPO of conformal dimension $\Delta=1$, linearized Einstein equations and asymptotic expansion of the LLM solutions upto the linear order were sufficient to read the vev. In that case, the KK maps between the 4dimensional fields and 11-dimensional fields are trivial. In this paper, we extend to the case of CPO of conformal dimension $\Delta=2$, which requires non-linear KK maps. We start with the compactification on $S^{7} / \mathbb{Z}_{k}$ of the 11-dimensional gravity equations in which the dynamical fields are written as a sum of the $\operatorname{AdS}_{4} \times S^{7} / \mathbb{Z}_{k}$ background and fluctuations. To obtain the vevs of the CPO of conformal dimension $\Delta=2$, it is sufficient to write the equations of motion up to the quadratic terms in fluctuations. After some manipulations for equations of gauge invariant fluctuation modes, we find that the quadratic terms contain higher derivatives, and thus we need to introduce some non-trivial field redefinitions (the KK maps) to obtain the canonical equations of motion for the 4-dimensional fields. The asymptotically $\mathrm{AdS}_{4}$ solutions to the resulting 4-dimensional equations of motion are obtained from the asymptotic expansion of the LLM solutions and combining various fields in the expansion, according to our well established non-linear KK maps. Using the holographic renormalization and asymptotic expansion of the LLM geometries, we read the vevs of the CPO of conformal dimension $\Delta=2$ and also confirm that the vevs of some massive $\mathrm{KK}$ graviton modes are vanishing as required by supersymmetry. ${ }^{1}$ On the field theory side, we use the discrete Higgs vacua of the mABJM theory to determine the $v e v$ of the CPO of conformal dimension $\Delta=2$ in the large $N$ limit. Due to computational difficulty, we focus on the case of the LLM solutions which are represented by squareshaped Young diagrams and we show that, in the large $N$ limit and finite $k$, the vev obtained from both the field theory side and the gravity side is $\left\langle\mathcal{O}^{(\Delta=2)}\right\rangle=\sqrt{k} N^{\frac{3}{2}} f_{(\Delta=2)}$, where $f_{\Delta}$ is independent of $N$.

The remaining part of the paper is organized as follows. In Sect. 2, we apply the KK reduction to 11-dimensional supergravity equations and obtain the equations for 4-dimensional gauge invariant fields. We also establish the non-trivial KK maps for some 4-dimensional gauge invariant fields. In Sect. 3, we obtain the CPO of conformal dimension $\Delta=2$ in the $\mathrm{mABJM}$ theory and determine its vev from the discrete Higgs vacua. In Sect. 4, we rearrange the asymptotic expansion of the LLM solutions according to our KK maps to obtain the asymptotically $\mathrm{AdS}_{4}$ solutions of the 4dimensional gravity equations of motion. From these solutions, we read the vevs of various 4-dimensional KK modes, using the gauge/gravity dictionary. In Sect. 5, we compare the gravity and the field theory results for the vevs of the CPOs

\footnotetext{
${ }^{1}$ See [16] for results of zeroth KK graviton modes.
}

and determine the values of some normalization factors. In Sect. 6, we draw our conclusions. In the Appendix, we give some details about the construction of CPO of conformal dimension $\Delta=2$.

\section{KK reduction of 11-dimensional gravity}

In this section, we discuss the compactification of 11dimensional gravity on $S^{7} / \mathbb{Z}_{k}$. The compactification involves expansion of the 11-dimensional fluctuations in terms of the spherical harmonics on $S^{7} / \mathbb{Z}_{k}$ and then projecting the equations of motions on those spherical harmonics to obtain the equations of motion for various KK modes. The resulting equations contain higher derivatives of those $\mathrm{KK}$ modes and the necessary KK maps are introduced for obtaining the canonical equations of motion of the 4-dimensional dynamical fields. The non-linear KK maps established in this section are used in Sect. 4 to obtain the asymptotically $\mathrm{AdS}_{4}$ solutions of 4-dimensional gravity equations of motion from the KK reduction of the LLM solutions. The vev of the CPO of conformal dimension $\Delta=2$ is read from such asymptotic solutions.

\subsection{Field equations at quadratic order}

In [16], we have written the 11-dimensional gravity equations of motion up to quadratic order in the fluctuations by perturbing the fields around the $\mathrm{AdS}_{4} \times S^{7} / \mathbb{Z}_{k}$ background as

$\mathbf{g}_{p q}=g_{p q}+h_{p q}, \quad \mathbf{F}_{p q r s}=F_{p q r s}+f_{p q r s}$,

where $p, q, \cdots=0, \cdots, 10$. For clarity, we summarize those quadratic order equations. The quadratic order equations are obtained by inserting (2.1) into the 11-dimensional gravity equations of motion and keeping all the terms up to quadratic order in the fluctuations $h_{p q}$ and $f_{p q r s}$. The results are

$$
\begin{aligned}
& \nabla^{r} \nabla_{p} h_{q r}+\nabla^{r} \nabla_{q} h_{p r}-\nabla^{2} h_{p q}-\nabla_{q} \nabla_{p} h_{r}^{r}-R h_{p q} \\
& \quad-g_{p q}\left(-R^{r s} h_{r s}+\nabla^{r} \nabla^{s} h_{r s}-\nabla^{2} h^{r}{ }_{r}\right) \\
& \quad+\frac{1}{48}\left(F_{r s t u} F^{r s t u} h_{p q}-4 g_{p q} h_{r s} F^{r}{ }_{t u v} F^{s t u v}\right) \\
& \quad+\frac{1}{24} g_{p q} f_{r s t u} F^{r s t u}-\frac{1}{2} h_{r s} F_{p t u}^{r} F_{q}{ }^{\text {stu }} \\
& \quad-\frac{1}{6}\left(f_{p r s t} F_{q}^{r s t}+F_{p r s t} f_{q}^{r s t}\right)+Q_{p q}=0, \\
& \nabla_{p}\left(h^{t}{ }_{t} F^{p q r s}\right)+2 \nabla_{p}\left(4 F_{t}{ }^{[p q r} h^{s] t}+f^{p q r s}\right) \\
& \quad+\frac{2}{\sqrt{-g}} \frac{1}{(4 !)^{2}} \tilde{\epsilon}^{p_{1} \cdots p_{4} q_{1} \cdots q_{4} q r s} f_{p_{1} \cdots p_{4}} F_{q_{1} \cdots q_{4}}+P^{q r s}=0,
\end{aligned}
$$


where the indices are raised (lowered) by the $\mathrm{AdS}_{4} \times S^{7} / \mathbb{Z}_{k}$ metric and the covariant derivatives are also those of the background. Here, $P^{q r s}$ and $Q_{p q}$ denote the quadratic terms in the fluctuations and are given by

$$
\begin{aligned}
P^{q r s}= & -\frac{1}{2} \nabla_{p}\left[\left(h_{t u} h^{t u}-\frac{1}{2}\left(h^{t}{ }_{t}\right)^{2}\right) F^{p q r s}\right] \\
& -8 \nabla_{p}\left(F_{u}{ }^{[p q r} h^{s] t} h_{t}{ }^{u}-\frac{3}{2} F^{t u[p q} h^{r}{ }_{t}{ }^{s]}{ }_{u}-f_{t}{ }^{[p q r} h^{s] t}\right) \\
& +\nabla_{p}\left[h^{t}{ }_{t}\left(4 F_{u}{ }^{[p q r} h^{s] u}+f^{p q r s}\right)\right] \\
& +\frac{1}{\sqrt{-g}} \frac{1}{(4 !)^{2}} \tilde{\epsilon}^{p_{1} \cdots p_{4} q_{1} \cdots q_{4} q r s} f_{p_{1} \cdots p_{4}} f_{q_{1} \cdots q_{4},} \\
Q_{p q}= & -\nabla_{r}\left[h^{r s}\left(\nabla_{p} h_{s q}+\nabla_{q} h_{s p}-\nabla_{s} h_{p q}\right)\right] \\
& +\frac{1}{2} \nabla_{p} h^{r s} \nabla_{q} h_{r s}+h^{r s} \nabla_{p} \nabla_{q} h_{r s} \\
& +\frac{1}{2} \nabla^{r} h^{s}{ }_{s}\left(\nabla_{p} h_{r q}+\nabla_{q} h_{r p}-\nabla_{r} h_{p q}\right) \\
& +\nabla^{r} h^{s}{ }_{p} \nabla_{r} h_{s q}-\nabla^{r} h^{s}{ }_{p} \nabla_{s} h_{q r}-g_{p q} R_{r s} h^{r t} h^{s}{ }_{t} \\
& +\frac{1}{2} g_{p q} \nabla_{r}\left[h^{r s}\left(2 \nabla^{t} h_{s t}-\nabla_{s} h^{t}{ }_{t}\right)\right] \\
& -\frac{3}{4} g_{p q} \nabla^{t} h^{r s} \nabla_{t} h_{r s}+\frac{1}{2} g_{p q} \nabla^{r} h^{s t} \nabla_{s} h_{t r} \\
& -\frac{1}{2} g_{p q} h^{r s} \nabla^{2} h_{r s} \\
& -\frac{1}{4} g_{p q} \nabla^{r} h^{s}{ }_{s}\left(2 \nabla^{t} h_{r t}-\nabla_{r} h^{t}{ }_{t}\right)+\frac{1}{2} g_{p q} h^{r s} \\
& \times\left(\nabla^{t} \nabla_{r} h_{t s}+\nabla^{t} \nabla_{s} h_{t r}-\nabla^{2} h_{r s}-\nabla_{r} \nabla_{s} h^{t}{ }_{t}\right) \\
& +h_{p q} h^{r s} R_{r s}-h_{p q}\left(\nabla^{r} \nabla^{s} h_{r s}-\nabla^{2} h^{r}{ }_{r}\right) \\
& +\frac{1}{12}\left[g_{p q} F_{r s t u} F^{r s t}{ }_{w} h^{u v} h_{v}{ }^{w}-g_{p q} F_{r s t u} f^{r s t}{ }_{v} h^{u v}\right. \\
& +\frac{3}{2} g_{p q} F_{r s t u} F^{r s}{ }_{v w} h^{t v} h^{u w}+\frac{1}{2} h_{p q} f_{r s t u} F^{r s t u} \\
& -h_{p q} F_{r s t u} F^{r s t}{ }_{v} h^{u v}+\frac{1}{4} g_{p q} f_{r s t u} f^{r s t u} \\
& \left.-g_{p q} f_{r s t u} F^{r s t}{ }_{v} h^{u v}\right]-\frac{1}{2}\left[F_{p s t u} F_{q}{ }^{s t}{ }_{w} h^{u v} h_{v}{ }^{w}\right. \\
& +F_{p s t u} F_{q}{ }^{s}{ }_{v w} h^{t v} h^{u w}-F_{p s t u} f_{q}{ }^{s t}{ }_{v} h^{u v} \\
& \left.-f_{p s t u} F_{q}{ }^{s t}{ }_{v} h^{u v}+\frac{1}{3} f_{p s t u} f_{q}{ }^{s t u}\right] . \\
& \\
&
\end{aligned}
$$

tions from the $\mathrm{AdS}_{4} \times S^{7} / \mathbb{Z}_{k}$ solutions. Keeping in mind the $\mathrm{SO}(2,1) \times \mathrm{SO}(4) / \mathbb{Z}_{k} \times \mathrm{SO}(4) / \mathbb{Z}_{k}$ isometry of the LLM solutions, we consider expansions in terms of the spherical harmonics with $\mathrm{SO}(4) / \mathbb{Z}_{k} \times \mathrm{SO}(4) / \mathbb{Z}_{k}$ symmetry. Since those spherical harmonics depend only on the $\tau$ coordinate, they are not affected by the orbifolding. This implies that expansions of the fluctuations $h_{p q}$ and $f_{p q r s}$ in terms of these spherical harmonics are the same, irrespective of the orbifolding. Therefore, in this section, we treat the expansion of the fluctuations $h_{p q}$ and $f_{p q r s}$ in terms of the spherical harmonics on $S^{7}$ and reintroduce the Chern-Simons level $k$ in the following sections.

In $[9,10]$, we have written a complete form of these expansions whereas we have argued in [16] that many of the KK modes do not contribute to the equations of motion at quadratic order. Therefore, we use the following truncated expansions,

$$
\begin{aligned}
h_{\mu \nu}(x, y) & =h_{\mu \nu}^{I_{1}}(x) Y^{I_{1}}(y), \quad h_{\rho}^{\rho}(x, y)=h^{I_{1}}(x) Y^{I_{1}}(y), \\
h_{(a b)} & =s^{I_{1}}(x) \nabla_{(a} \nabla_{b)} Y^{I_{1}}(y), \quad h_{a}^{a}(x, y)=\phi^{I_{1}}(x) Y^{I_{1}}(y), \\
f_{\mu \nu \rho \sigma}(x, y) & =\frac{2}{3} \nabla_{[\mu} t^{\lambda I_{1}}(x) \epsilon_{\nu \rho \sigma] \lambda} Y^{I_{1}}(y), \\
f_{\mu \nu \rho a}(x, y) & =-\frac{1}{3 !} \epsilon_{\mu \nu \rho}{ }^{\sigma} t_{\sigma}^{I_{1}}(x) \nabla_{a} Y^{I_{1}}(y), \\
f_{\mu a b c}(x, y) & =\nabla_{\mu} t^{I_{35}}(x) Y_{a b c}^{I_{35}}(y), \\
f_{a b c d}(x, y) & =4 t^{I_{35}}(x) \nabla_{[a} Y_{b c d]}^{I_{35}}(y),
\end{aligned}
$$

where $I_{n}=0,1,2, \ldots$, we have split the 11-dimensional indices into the $\mathrm{AdS}_{4}$ indices $(\mu, v, \cdots=0, \cdots, 3)$ and the $S^{7}$ indices $(a, b, \cdots=4, \cdots, 10), x$ denotes the $\mathrm{AdS}_{4}$ coordinates and $y$ denotes the $S^{7}$ coordinates. The notation $(a b)$ means symmetrized traceless combination, while $[a b \cdots]$ denotes complete antisymmetrization of indices. Here, $Y^{I_{1}}$ and $Y_{a b c}^{I_{35}}$ are the scalar and antisymmetric 3-tensor spherical harmonics on $S^{7}$, respectively.

Plugging (2.7) into the ( $\mu \nu)$ component of (2.2) and then projecting on the scalar spherical harmonics $Y^{I_{1}}$, we obtain

$$
\begin{aligned}
& -\left(\square+\Lambda^{I_{1}}-\frac{24}{L^{2}}\right) h_{\mu \nu}^{I_{1}}+\nabla^{\rho} \nabla_{\mu} h_{\nu \rho}^{I_{1}}+\nabla^{\rho} \nabla_{\nu} h_{\mu \rho}^{I_{1}} \\
& -\nabla_{\mu} \nabla_{\nu}\left(h^{I_{1}}+\phi^{I_{1}}\right) \\
& +g_{\mu \nu}\left(\square+\Lambda^{I_{1}}-\frac{30}{L^{2}}\right) h^{I_{1}}+g_{\mu \nu}\left(\square+\frac{6}{7} \Lambda^{I_{1}}+\frac{6}{L^{2}}\right) \phi^{I_{1}} \\
& -g_{\mu \nu}\left(\frac{6}{7} \Lambda^{I_{1}}+\frac{6}{L^{2}}\right) \Lambda^{I_{1}} s^{I_{1}} \\
& -g_{\mu \nu} \nabla^{\rho} \nabla^{\sigma} h_{\rho \sigma}^{I_{1}}+\frac{1}{L} g_{\mu \nu} \nabla^{\rho} t_{\rho}^{I_{1}}+2 Q_{\mu \nu}^{I_{1}}=0,
\end{aligned}
$$

where $L$ is the radius of $S^{7}$. Later, we will identify the fluctuations $h_{p q}$ and $f_{p q r s}$ with the deviations of the LLM solu- where $\square \equiv \nabla_{\mu} \nabla^{\mu}, L$ is the radius of $S^{7}, Q_{\mu \nu}^{I_{1}}=$ $\frac{1}{\omega_{7}} \int_{S^{7}} \frac{1}{2} Q_{\mu \nu} Y^{I_{1}}$, and $\Lambda^{I_{1}}=-\frac{I_{1}\left(I_{1}+6\right)}{L^{2}}$ is the eigenvalue corresponding to the scalar harmonics $Y^{I_{1}}$. The trace of the above equation leads to 


$$
\begin{aligned}
& \left(\square+\frac{3}{2} \Lambda^{I_{1}}-\frac{48}{L^{2}}\right) h^{I_{1}}-\nabla^{\mu} \nabla^{v} h_{\mu \nu} \\
& +\frac{3}{2}\left(\square+\frac{8}{7} \Lambda^{I_{1}}+\frac{8}{L^{2}}\right) \phi^{I_{1}}+\frac{2}{L} \nabla^{\rho} t_{\rho}^{I_{1}} \\
& -12 \Lambda^{I_{1}}\left(\frac{1}{7} \Lambda^{I_{1}}+\frac{1}{L^{2}}\right) s^{I_{1}}+Q_{h}^{I_{1}}=0,
\end{aligned}
$$

where $Q_{h}^{I_{1}}=g^{\mu \nu} Q_{\mu \nu}^{I_{1}}$. Secondly, projecting the $(\mu a)$ component of (2.2) on $\nabla^{a} Y^{I_{1}}\left(I_{1} \neq 0\right),{ }^{2}$ we obtain

$$
\begin{aligned}
& -\left(\frac{6}{7} \Lambda^{I_{1}}+\frac{6}{L^{2}}\right) \nabla_{\mu} s^{I_{1}}+\frac{6}{7} \nabla_{\mu} \phi^{I_{1}}-\nabla^{v} h_{\mu \nu}^{I_{1}}+\nabla_{\mu} h^{I_{1}} \\
& -\frac{1}{L} t_{\mu}^{I_{1}}+Q_{\mu}^{I_{1}}=0
\end{aligned}
$$

where $Q_{\mu}^{I_{1}}=\frac{1}{\omega_{7}} \int_{S^{7}} Q_{\mu a} \nabla^{a} Y^{I_{1}}$. Thirdly, projecting the $(a b)$ component of (2.2) on $g^{a b} Y^{I_{1}}$ and $\nabla^{(a} \nabla^{b)} Y^{I_{1}}\left(I_{1} \neq 0\right)$, we obtain two scalar equations

$$
\begin{aligned}
& 3\left(\square+\frac{5}{7} \Lambda^{I_{1}}+\frac{5}{L^{2}}\right) \phi^{I_{1}}+\frac{7}{2}\left(\square+\frac{6}{7} \Lambda^{I_{1}}+\frac{6}{L^{2}}\right) h^{I_{1}} \\
& -\frac{7}{2} \nabla^{\mu} \nabla^{v} h_{\mu \nu}^{I_{1}}-\frac{7}{2 L} \nabla^{\rho} t_{\rho}^{I_{1}} \\
& \quad-15 \Lambda^{I_{1}}\left(\frac{\Lambda^{I_{1}}}{7}+\frac{1}{L^{2}}\right) s^{I_{1}}+Q_{\phi}^{I_{1}}=0, \\
& \Lambda^{I_{1}}\left\{\left(\square-\frac{5}{7} \Lambda^{I_{1}}\right) s^{I_{1}}+h^{I_{1}}+\frac{5}{7} \phi^{I_{1}}\right\}-Q_{s}^{I_{1}}=0,
\end{aligned}
$$

where $Q_{\phi}^{I_{1}}=\frac{1}{\omega 7} \int_{S^{7}} \frac{1}{2} Q_{a b} g^{a b} Y^{I_{1}}$ and $Q_{S}^{I_{1}}=\frac{1}{7}\left(6 \Lambda^{I_{1}}+\right.$ $\left.\frac{42}{L^{2}}\right)^{-1} \frac{1}{\omega_{7}} \int_{S^{7}} Q_{a b} \nabla^{(a} \nabla^{b)} Y^{I_{1}}$. Similarly, inserting (2.7) into $(\mu \nu \rho)$ component of (2.3) and projecting on $Y^{I_{1}}$, we obtain the following equation ${ }^{3}$

$$
\begin{aligned}
& \frac{2}{3} \nabla^{\sigma} \nabla_{[\sigma} t^{\lambda I_{1}} \epsilon_{\mu \nu \rho] \lambda}+\frac{\Lambda^{I_{1}}}{3 !} \epsilon_{\mu \nu \rho}{ }^{\sigma} t_{\sigma}^{I_{1}}-\frac{3}{L} \epsilon_{\sigma \mu \nu \rho} \nabla^{\sigma}\left(h^{I_{1}}+\phi^{I_{1}}\right) \\
& -\frac{24}{L} \nabla^{\sigma} h_{\lambda[\sigma}^{I_{1}} \epsilon_{\mu \nu \rho]}{ }^{\lambda}+P_{\mu \nu \rho}^{I_{1}}=0
\end{aligned}
$$

where $P_{\mu \nu \rho}^{I_{1}}=\frac{1}{\omega 7} \int_{S^{7}} P_{\mu \nu \rho} Y^{I_{1}}$. Applying $\epsilon_{\mu^{\prime}}^{\mu \nu \rho} \nabla_{\nu^{\prime}}$ to (2.13), we obtain

$$
\begin{aligned}
& -\frac{18}{\Lambda^{I_{1}}} \nabla_{\mu} \nabla_{\nu}\left(-h^{I_{1}}+\phi^{I_{1}}\right)-L \nabla_{\nu} t_{\mu}^{I_{1}}-\frac{L}{\Lambda^{I_{1}}} \nabla_{\mu} \nabla_{\nu} \nabla^{\rho} t_{\rho}^{I_{1}} \\
& \quad+\tilde{Q}_{\mu \nu}^{I_{1}}=0
\end{aligned}
$$

\footnotetext{
${ }^{2}$ See [16] for the zeroth mode results.

${ }^{3}$ More equations can be obtained by projecting the ( $\left.\mu \nu a, \mu a b, a b c\right)$ components of (2.4) on appropriate spherical harmonic elements, however those equations are not required for our purpose here. See [16] for the full list of equations.
}

where $\tilde{Q}_{\mu \nu}^{I_{1}}=-\frac{L}{\Lambda^{I_{1}}} \epsilon_{\mu}{ }^{\rho \sigma \lambda} \nabla_{\nu} P_{\rho \sigma \lambda}^{I_{1}}$. The trace of the above equation gives

$\frac{18}{L} \square\left(-h^{I_{1}}+\phi^{I_{1}}\right)+\left(\square+\Lambda^{I_{1}}\right) \nabla^{\rho} t_{\rho}^{I_{1}}+Q_{\psi}^{I_{1}}=0$,

where $Q_{\psi}^{I_{1}}=-\Lambda^{I_{1}} g^{\mu \nu} \tilde{Q}_{\mu \nu}^{I_{1}}$.

\subsection{Quadratic order equations for KK modes}

The quadratic order equations we listed in the previous subsection lead to the quadratic order equations of motions for various 4-dimensional gauge invariant KK modes. In general, the 4-dimensional gravity spectrum, which is obtained from the KK reduction of the 11-dimensional gravity, is composed of three towers of scalar modes, two towers of pseudoscalar modes, two towers of vector modes, one tower of pseudovector modes, and one tower of spin-two modes $[9,10]$. Here, we follow the gauge choice of the LLM solutions in which $h_{\mu a}$ and $f_{\mu \nu a b}$ are zero and as a result some of the KK towers are absent. In addition, in this paper, we are interested in the gravity field which is dual to the CPO of conformal dimension $\Delta=2$ in the mABJM theory. Such dual gravity field is a part of the three KK towers of scalar modes with $I_{1}=4$. Therefore, from now on we focus on the equations of motion for the KK modes with $I_{1}=4$.

Setting $I_{1}=4$ in (2.8)-(2.15) and rearranging the equations, we obtain the following set of equations,

$$
\begin{aligned}
\square \hat{h}_{\mu \nu}^{4}= & \frac{32}{L^{2}} \hat{h}_{\mu \nu}^{4}+\frac{1}{20} \nabla_{\mu} \nabla_{\nu} \hat{\psi}^{4}-\frac{9}{10} \nabla_{\mu} \nabla_{\nu} \hat{\phi}^{4}-\frac{4}{3 L^{2}} g_{\mu \nu} \hat{\psi}^{4} \\
& -\frac{40}{7 L^{2}} g_{\mu \nu} \hat{\phi}^{4}+\nabla_{\mu} Q_{\nu}^{4}+\nabla_{\nu} Q_{\mu}^{4} \\
& +\frac{L^{2}}{40} \nabla_{\mu} \nabla_{\nu} Q_{s}^{4}-\frac{2}{9} g_{\mu \nu}\left(Q_{h}^{4}+Q_{\phi}^{4}-\frac{9}{10} Q_{s}^{4}\right) \\
& -\frac{1}{L^{2}}\left(\tilde{Q}_{\mu \nu}^{4}+\tilde{Q}_{\nu \mu}^{4}\right)+2 Q_{\mu \nu}^{4}, \\
\hat{J}_{\mu \nu}^{4}= & -\frac{L^{2}}{40} \nabla_{\mu} \nabla_{\nu} \hat{\psi}^{4}+\frac{9 L^{2}}{20} \nabla_{\mu} \nabla_{\nu} \hat{\phi}^{4}+\frac{1}{2}\left(\tilde{Q}_{\mu \nu}^{4}+\tilde{Q}_{\nu \mu}^{4}\right), \\
\square \hat{\phi}^{4}= & \frac{28}{L^{2}} \hat{\phi}^{4}+\frac{14}{3 L^{2}} \hat{\psi}^{4}-\frac{14}{9} Q_{h}^{4}+\frac{4}{9} Q_{\phi}^{4}-Q_{s}^{4}, \\
\square \hat{\psi}^{4}= & \frac{124}{L^{2}} \hat{\psi}^{4}+\frac{7128}{7 L^{2}} \hat{\phi}^{4}-28 Q_{h}^{4}+8 Q_{\phi}^{4}+Q_{\psi}^{4},
\end{aligned}
$$

where we have introduced $u_{\mu \nu}^{I_{1}} \equiv \frac{L}{2}\left(\nabla_{\mu} t_{\nu}^{I_{1}}+\nabla_{\nu} t_{\mu}^{I_{1}}\right), u^{I_{1}} \equiv$ $g^{\mu v} u_{\mu \nu}^{I_{1}}$, and the following gauge invariant combinations,

$$
\begin{array}{rlrl}
\hat{h}_{\mu \nu}^{I_{1}} & \equiv h_{\mu \nu}^{I_{1}}+\nabla_{\mu} \nabla_{\nu} S^{I_{1}}, & \hat{J}_{\mu \nu}^{I_{1}} \equiv u_{\mu \nu}^{I_{1}}+18 \nabla_{\mu} \nabla_{\nu} S^{I_{1}}, \\
\hat{\phi}^{I_{1}} \equiv \phi^{I_{1}}-\Lambda^{I_{1}} s^{I_{1}}, & \hat{\psi}^{I_{1}} \equiv 18 h^{I_{1}}-u^{I_{1}} .
\end{array}
$$




\subsubsection{Spin-zero field equations}

The equations of motion for spin-zero mass eigenstates are given by the linear combinations of (2.18) and (2.19). Introducing the mass eigenstates

$\check{\phi}^{4}=\frac{297}{49} \hat{\phi}^{4}+\frac{11}{14} \hat{\psi}^{4}, \quad \check{\psi}^{4}=-\frac{297}{49} \hat{\phi}^{4}+\frac{3}{14} \hat{\psi}^{4}$,

and combining (2.18) and (2.19), we obtain the following diagonalized equations

$$
\begin{aligned}
& \left(\square+\frac{8}{L^{2}}\right) \check{\psi}^{4}-\frac{24}{7} Q_{h}^{4}+\frac{48}{49} Q_{\phi}^{4}-\frac{3}{14} Q_{\psi}^{4}+\frac{297}{49} Q_{s}^{4}=0, \\
& \left(\square-\frac{160}{L^{2}}\right) \check{\phi}^{4}+\frac{220}{7} Q_{h}^{4}-\frac{440}{49} Q_{\phi}^{4}-\frac{11}{14} Q_{\psi}^{4}-\frac{297}{49} Q_{s}^{4}=0 .
\end{aligned}
$$

All the quadratic terms in the above equations are composed of the expressions which are quadratic in the fields $h_{\mu \nu}^{I_{1}}, t_{\mu}^{I_{1}}, h^{I_{1}}, \phi^{I_{1}}, u^{I_{1}}, s^{I_{1}}, t^{I_{35}}$ and their derivatives, with infinite summations over $I_{1}$ and $I_{35}$. The LLM solution solves the 11-dimensional equations of motion order by order in the mass parameter $\mu_{0}$ of the LLM geometries $[9,10,16]$. In the above equations of motion, we have kept only up to the quadratic terms in the fluctuations and they are expected to be solved by the LLM solution only up to quadratic order in $\mu_{0}$. On the other hand, except for the modes with $I_{1}=2$ and $I_{35}=1$, the asymptotic expansions of the other modes are non-linear in the expansion parameter $\mu_{0}$. Thus, the relevant quadratic terms in the above equations are built only by the modes with $I_{1}=2$ and $I_{35}=1$. In addition, we note that for the spherical harmonics on $S^{7}$ with $\mathrm{SO}(4) \times \mathrm{SO}(4)$ symmetry, (see $[9,10])$

$$
\begin{gathered}
\int_{S^{7}} Y^{4} Y_{a b c}^{1} g^{a a^{\prime}} g^{b b^{\prime}} g^{c c^{\prime}} Y_{a^{\prime} b^{\prime} c^{\prime}}^{1}=0, \\
\int_{S^{7}} \nabla^{a} \nabla^{b} Y^{4} Y_{a c d}^{1} g^{c c^{\prime}} g^{d d^{\prime}} Y_{b c^{\prime} d^{\prime}}^{1}=0 .
\end{gathered}
$$

The LLM solutions depend only on such spherical harmonics. In that case, the terms involving $t^{I_{35}=1}$ are also absent and the quadratic terms depend only on $h_{\mu \nu}^{2}, t_{\mu}^{2}, h^{2}, \phi^{2}, u^{2}, s^{2}$ and their derivatives. Combining the four scalar fields $h^{2}, \phi^{2}, u^{2}, s^{2}$, we obtain two gauge invariant physical mode, $\breve{\phi}^{2}=\frac{9}{70}\left(7 \hat{\psi}^{2}+18 \hat{\phi}^{2}\right), \check{\psi}^{2}=\frac{1}{70}\left(7 \hat{\psi}^{2}-162 \hat{\phi}^{2}\right)$, which are mass eigenstates. The other potentially relevant gauge invariant physical mode is the second KK massive graviton mode, which is given by

$\check{h}_{(\mu \nu)}^{2}=\hat{h}_{(\mu \nu)}^{2}-\frac{1}{4} \hat{J}_{(\mu \nu)}^{2}+\frac{15 L^{2}}{112} \nabla_{(\mu} \nabla_{\nu)} \hat{\phi}^{2}-\frac{L^{2}}{96} \nabla_{(\mu} \nabla_{\nu)} \hat{\psi}^{2}$.
In general, our quadratic terms depend on the two physical scalar modes $\left(\breve{\phi}^{2}, \breve{\psi}^{2}\right)$ and the second KK massive graviton mode $\breve{h}_{(\mu \nu)}^{2}$. However, the leading order terms in the asymptotic expansions of $\breve{h}_{(\mu \nu)}^{2}$ and $\check{\phi}^{2}$ are $\mu_{0}^{3}$-order, and they are irrelevant for quadratic order equations. As a result, the otherwise very complex quadratic terms are composed of only $\breve{\psi}^{2}$, and are given by

$$
\begin{aligned}
& Q_{h}^{4}=-\frac{1}{41472 \sqrt{10} L^{2}}\left(41216 \breve{\psi}^{2} \breve{\psi}^{2}\right. \\
& +2560 L^{2} \nabla_{\rho} \check{\psi}^{2} \nabla^{\rho} \breve{\psi}^{2}+88 L^{4} \nabla_{\rho} \nabla_{\sigma} \breve{\psi}^{2} \nabla^{\rho} \nabla^{\sigma} \breve{\psi}^{2} \\
& \left.-\nabla_{\rho} \nabla_{\sigma} \nabla_{\lambda} \check{\psi}^{2} \nabla^{\rho} \nabla^{\sigma} \nabla^{\lambda} \breve{\psi}^{2}\right) \text {, } \\
& Q_{\phi}^{4}=-\frac{1}{82944 \sqrt{10} L^{2}}\left(126080 \breve{\psi}^{2} \breve{\psi}^{2}\right. \\
& +12736 L^{2} \nabla_{\rho} \check{\psi}^{2} \nabla^{\rho} \breve{\psi}^{2}-32 L^{4} \nabla_{\rho} \nabla_{\sigma} \breve{\psi}^{2} \nabla^{\rho} \nabla^{\sigma} \breve{\psi}^{2} \\
& \left.-7 L^{6} \nabla_{\rho} \nabla_{\sigma} \nabla_{\lambda} \check{\psi}^{2} \nabla^{\rho} \nabla^{\sigma} \nabla^{\lambda} \breve{\psi}^{2}\right) \text {, } \\
& Q_{s}^{4}=-\frac{5}{1944 \sqrt{10} L^{2}}\left(120 \check{\psi}^{2} \check{\psi}^{2}+8 L^{2} \nabla_{\rho} \check{\psi}^{2} \nabla^{\rho} \check{\psi}^{2}\right. \\
& \left.+L^{4} \nabla_{\rho} \nabla_{\sigma} \check{\psi}^{2} \nabla^{\rho} \nabla^{\sigma} \check{\psi}^{2}\right) \text {, } \\
& Q_{\psi}^{4}=-\frac{1}{576 \sqrt{10} L^{2}}\left(3584 \breve{\psi}^{2} \breve{\psi}^{2}+416 L^{2} \nabla_{\rho} \breve{\psi}^{2} \nabla^{\rho} \breve{\psi}^{2}\right. \\
& \left.-80 L^{4} \nabla_{\rho} \nabla_{\sigma} \breve{\psi}^{2} \nabla^{\rho} \nabla^{\sigma} \breve{\psi}^{2}\right) \\
& +L^{6} \nabla_{\rho} \nabla_{\sigma} \nabla_{\lambda} \check{\psi}^{2} \nabla^{\rho} \nabla^{\sigma} \nabla^{\lambda} \breve{\psi}^{2}, \\
& Q_{\mu \nu}^{4}=-\frac{1}{41472 \sqrt{10} L^{2}}\left[\frac { 1 } { 2 } g _ { \mu \nu } \left(22400 \breve{\psi}^{2} \breve{\psi}^{2}\right.\right. \\
& +1600 L^{2} \nabla_{\rho} \breve{\psi}^{2} \nabla^{\rho} \breve{\psi}^{2}+48 L^{4} \nabla_{\rho} \nabla_{\sigma} \breve{\psi}^{2} \nabla^{\rho} \nabla^{\sigma} \breve{\psi}^{2} \\
& \left.-L^{4} \nabla_{\rho} \nabla_{\sigma} \nabla_{\lambda} \check{\psi}^{2} \nabla^{\rho} \nabla^{\sigma} \nabla^{\lambda} \check{\psi}^{2}\right) \\
& +320 \nabla_{\mu} \check{\psi}^{2} \nabla_{\nu} \breve{\psi}^{2}+448 L^{2} \breve{\psi}^{2} \nabla_{\mu} \nabla_{\nu} \breve{\psi}^{2} \\
& +48 L^{4} \nabla_{\rho} \check{\psi}^{2} \nabla_{\mu} \nabla_{\nu} \nabla^{\rho} \breve{\psi}^{2}-8 L^{6} \nabla_{\mu} \nabla_{\rho} \check{\psi}^{2} \nabla_{\nu} \nabla^{\rho} \check{\psi}^{2} \\
& \left.+L^{6} \nabla_{\mu} \nabla_{\rho} \nabla_{\sigma} \check{\psi}^{2} \nabla_{\nu} \nabla^{\rho} \nabla^{\sigma} \breve{\psi}^{2}\right] \text {, } \\
& Q_{\mu}^{4}=\frac{1}{41472 \sqrt{10}}\left(1568 \check{\psi}^{2} \nabla_{\mu} \check{\psi}^{2}+24 L^{2} \nabla_{\rho} \check{\psi}^{2} \nabla_{\mu} \nabla^{\rho} \check{\psi}^{2}\right. \\
& \left.+L^{4} \nabla_{\rho} \nabla_{\sigma} \breve{\psi}^{2} \nabla_{\mu} \nabla^{\rho} \nabla^{\sigma} \breve{\psi}^{2}\right) \text {, } \\
& \tilde{Q}_{\mu \nu}^{4}=\frac{L^{2}}{23040 \sqrt{10}}\left(384 \nabla_{\mu} \check{\psi}^{2} \nabla_{\nu} \check{\psi}^{2}+40 L^{2} \nabla_{\mu} \nabla_{\rho} \check{\psi}^{2} \nabla_{\nu}\right. \\
& \times \nabla^{\rho} \breve{\psi}^{2}-L^{4} \nabla_{\mu} \nabla_{\rho} \nabla_{\sigma} \check{\psi}^{2} \nabla_{\nu} \nabla^{\rho} \nabla^{\sigma} \check{\psi}^{2} \\
& +384 \breve{\psi}^{2} \nabla_{\mu} \nabla_{\nu} \check{\psi}^{2}+40 L^{2} \nabla_{\rho} \breve{\psi}^{2} \nabla_{\mu} \nabla_{\nu} \nabla^{\rho} \breve{\psi}^{2} \\
& \left.-L^{4} \nabla_{\rho} \nabla_{\sigma} \check{\psi}^{2} \nabla_{\mu} \nabla_{\nu} \nabla^{\rho} \nabla^{\sigma} \check{\psi}^{2}\right) \text {. }
\end{aligned}
$$


Inserting these quadratic terms into (2.22), we obtain

$$
\begin{aligned}
& \left(\square+\frac{8}{L^{2}}\right) \check{\psi}^{4}+\frac{1}{8064 \sqrt{10} L^{2}}\left(11136 \check{\psi}^{2} \breve{\psi}^{2}\right. \\
& \quad+736 L^{2} \nabla_{\rho} \check{\psi}^{2} \nabla^{\rho} \breve{\psi}^{2}-304 L^{4} \nabla_{\rho} \nabla_{\sigma} \check{\psi}^{2} \nabla^{\rho} \nabla^{\sigma} \breve{\psi}^{2} \\
& \left.\quad+3 L^{6} \nabla_{\rho} \nabla_{\sigma} \nabla_{\lambda} \check{\psi}^{2} \nabla^{\rho} \nabla^{\sigma} \nabla^{\lambda} \breve{\psi}^{2}\right)=0, \\
& \left(\square-\frac{160}{L^{2}}\right) \check{\phi}^{4}+\frac{11}{8064 \sqrt{10} L^{2}}\left(-7936 \check{\psi}^{2} \breve{\psi}^{2}\right. \\
& \quad+96 L^{2} \nabla_{\rho} \check{\psi}^{2} \nabla^{\rho} \breve{\psi}^{2}-120 L^{4} \nabla_{\rho} \nabla_{\sigma} \check{\psi}^{2} \nabla^{\rho} \nabla^{\sigma} \check{\psi}^{2} \\
& \left.\quad+L^{6} \nabla_{\rho} \nabla_{\sigma} \nabla_{\lambda} \check{\psi}^{2} \nabla^{\rho} \nabla^{\sigma} \nabla^{\lambda} \check{\psi}^{2}\right)=0 .
\end{aligned}
$$

This shows that the usual compactification of the 11dimensional supergravity on $S^{7}$ results in the field equations which contain higher derivative terms. In order to obtain the canonical 4-dimensional gravity equations of motion, we need to introduce some field redefinitions to absorb those higher derivative terms $[11,14-16]$. For instance, for some scalar KK mode whose equation of motion contains up to four derivatives, the field redefinition is of the form

$S^{I}=s^{I}+K_{I J_{1} J_{2}} t^{J_{1}} t^{J_{2}}+L_{I J_{1} J_{2}} \nabla_{\mu} t^{J_{1}} \nabla^{\mu} t^{J_{2}}$,

where $K_{I J_{1} J_{2}}, L_{I J_{1} J_{2}}$ are some numerical coefficients, $s^{I}$ represent a gauge invariant 11-dimensional field and $S^{I}$ is the corresponding 4-dimensional field. The $t^{J_{i}}$ 's represent the gauge invariant 11-dimensional fields that appear in higher derivative part of the equations of motion of $s^{I}$. In Sect. 5, we will use the asymptotic expansion of the 4-dimensional field $S^{I}$ to read the vev of the dual operator. Then, the nonlinearity of the field redefinition (2.27) shows that the vev gets contributions from terms which are non-linear in the 11-dimensional fields.

The 4-dimensional gravity equations of motion should read as follows,

$$
\begin{aligned}
& \left(\square+\frac{8}{L^{2}}\right) \Psi^{4}+\alpha \Psi^{2} \Psi^{2}=0, \\
& \left(\square-\frac{160}{L^{2}}\right) \Phi^{4}+\beta \Psi^{2} \Psi^{2}=0
\end{aligned}
$$

where $\Psi^{2} \equiv \check{\psi}^{2}$. Since the equations in (2.26) contain the terms with up to sextic derivatives, the field redefinitions absorbing those sextic derivatives should contain terms with up to quartic derivatives

$$
\begin{aligned}
\Psi^{4}= & \check{\psi}^{4}+A_{1} \check{\psi}^{2} \check{\psi}^{2}+A_{2} \nabla_{\rho} \check{\psi}^{2} \nabla^{\rho} \check{\psi}^{2} \\
& +A_{3} \nabla_{\rho} \nabla_{\sigma} \check{\psi}^{2} \nabla^{\rho} \nabla^{\sigma} \check{\psi}^{2}, \\
\Phi^{4}= & \check{\phi}^{4}+B_{1} \check{\psi}^{2} \check{\psi}^{2}+B_{2} \nabla_{\rho} \check{\psi}^{2} \nabla^{\rho} \breve{\psi}^{2} \\
& +B_{3} \nabla_{\rho} \nabla_{\sigma} \check{\psi}^{2} \nabla^{\rho} \nabla^{\sigma} \check{\psi}^{2} .
\end{aligned}
$$

Insertion of (2.29) into (2.28) and comparison with (2.26) fix the unknown coefficients in (2.28)-(2.29) as

$$
\begin{aligned}
& A_{1}=-\frac{25}{168 \sqrt{10}}, \quad A_{2}=-\frac{7 L^{2}}{576 \sqrt{10}}, \\
& A_{3}=\frac{L^{4}}{5376 \sqrt{10}}, \quad \alpha=0, \\
& B_{1}=\frac{11}{168 \sqrt{10}}, \quad B_{2}=0, \quad B_{3}=\frac{11 L^{4}}{5376 \sqrt{10}}, \quad \beta=0 .
\end{aligned}
$$

The field redefinition of the type (2.29) is usually called the KK map between the 11-dimensional fields $\left(\breve{\psi}^{4}, \breve{\phi}^{4}\right)$ and the 4-dimensional fields $\left(\Psi^{4}, \Phi^{4}\right)$.

\subsubsection{Spin-two field equations}

The equation of motion for the fourth KK massive graviton mode is a linear combinations of the Eqs. (2.16)-(2.19). Let us define the spin-two mass eigenstate as

$$
\begin{aligned}
\check{h}_{\mu \nu}^{4}= & \hat{h}_{\mu \nu}^{4}+a_{1} \hat{J}_{\mu \nu}^{4}+a_{2} \nabla_{\mu} \nabla_{\nu} \hat{\phi}^{4}+a_{3} \nabla_{\mu} \nabla_{\nu} \hat{\psi}^{4} \\
& +g_{\mu \nu}\left(c \hat{\phi}^{4}+d \hat{\psi}^{4}\right) \\
= & \hat{h}_{\mu \nu}^{4}+a \nabla_{\mu} \nabla_{\nu} \hat{\phi}^{4}+b \nabla_{\mu} \nabla_{\nu} \hat{\psi}^{4}+g_{\mu \nu}\left(c \hat{\phi}^{4}+d \hat{\psi}^{4}\right),
\end{aligned}
$$

where in the second line, we have used the algebraic equation (2.17) to eliminate $\hat{J}_{\mu \nu}^{4}$ up to a redundant quadratic term, which we omit from the definition. Organizing the Eqs. (2.16)-(2.19) according to this definition and setting

$a=-\frac{17 L^{2}}{1120}, \quad b=\frac{L^{2}}{2880}, \quad c=\frac{11}{56}, \quad d=\frac{1}{144}$,

we obtain the diagonalized equation for the mass eigenstate

$$
\begin{aligned}
\left(\square-\frac{32}{L^{2}}\right) \check{h}_{\mu \nu}^{4} & -\left[\nabla_{\mu} Q_{\nu}^{4}+\nabla_{\nu} Q_{\mu}^{4}+\frac{L^{2}}{72} \nabla_{\mu} \nabla_{\nu}\right. \\
& \times\left(Q_{h}^{4}+\frac{99}{140} Q_{s}^{4}-\frac{2}{7} Q_{\phi}^{4}+\frac{1}{40} Q_{\psi}^{4}\right) \\
& -g_{\mu \nu}\left(\frac{11}{18} Q_{h}^{4}-\frac{11}{40} Q_{s}^{4}+\frac{1}{9} Q_{\phi}^{4}-\frac{7}{720} Q_{\psi}^{4}\right) \\
& \left.-\frac{2}{L^{2}} \tilde{Q}_{\mu \nu}^{4}+2 Q_{\mu \nu}^{4}\right]=0 .
\end{aligned}
$$

Inserting the quadratic terms in (2.25) into this equation, we rewrite (2.33) as 


$$
\begin{aligned}
(\square & \left.-\frac{32}{L^{2}}\right) \check{h}_{\mu \nu}^{4}-\frac{1}{1080 \sqrt{10}} \\
& \times\left[2 g _ { \mu \nu } \left(\frac{49}{L^{2}} \check{\psi}^{2} \check{\psi}^{2}+\frac{137}{72} \nabla_{\rho} \check{\psi}^{2} \nabla^{\rho} \check{\psi}^{2}\right.\right. \\
& +\frac{115 L^{2}}{288} \nabla_{\rho} \nabla_{\sigma} \check{\psi}^{2} \nabla^{\rho} \nabla^{\sigma} \check{\psi}^{2} \\
& \left.-\frac{7 L^{4}}{768} \nabla_{\rho} \nabla_{\sigma} \nabla_{\lambda} \check{\psi}^{2} \nabla^{\rho} \nabla^{\sigma} \nabla^{\lambda} \check{\psi}^{2}\right)+\nabla_{\mu} \check{\psi}^{2} \nabla_{\nu} \check{\psi}^{2} \\
& +\frac{17}{3} \check{\psi}^{2} \nabla_{\mu} \nabla_{\nu} \check{\psi}^{2}-\frac{259 L^{2}}{72} \nabla_{\mu} \nabla_{\rho} \check{\psi}^{2} \nabla_{\nu} \nabla^{\rho} \check{\psi}^{2} \\
& -\frac{469 L^{2}}{72} \nabla_{\rho} \check{\psi}^{2} \nabla_{\mu} \nabla_{\nu} \nabla^{\rho} \check{\psi}^{2} \\
+ & \frac{11 L^{4}}{144} \nabla_{\mu} \nabla_{\rho} \nabla_{\sigma} \check{\psi}^{2} \nabla_{\nu} \nabla^{\rho} \nabla^{\sigma} \check{\psi}^{2} \\
+ & \frac{37 L^{4}}{288} \nabla_{\rho} \nabla_{\sigma} \check{\psi}^{2} \nabla_{\mu} \nabla_{\nu} \nabla^{\rho} \nabla^{\sigma} \check{\psi}^{2} \\
& -\frac{L^{6}}{768} \nabla_{\mu} \nabla_{\rho} \nabla_{\sigma} \nabla_{\lambda} \check{\psi}^{2} \nabla_{\nu} \nabla^{\rho} \nabla^{\sigma} \nabla^{\lambda} \check{\psi}^{2} \\
& \left.-\frac{L^{6}}{768} \nabla_{\rho} \nabla_{\sigma} \nabla_{\lambda} \check{\psi}^{2} \nabla_{\mu} \nabla_{\nu} \nabla^{\rho} \nabla^{\sigma} \nabla^{\lambda} \check{\psi}^{2}\right]=0 .
\end{aligned}
$$

This spin-two field equation contains the terms with up to octic derivatives. In order to absorb these higher derivative terms, we need to introduce another field redefinition with up to sextic derivatives as follows

$$
\begin{aligned}
H_{\mu \nu}^{4}= & \check{h}_{\mu \nu}^{4}+g_{\mu \nu}\left(\tilde{C}_{0} \check{\psi}^{2} \check{\psi}^{2}+\tilde{C}_{1} \nabla_{\rho} \check{\psi}^{2} \nabla^{\rho} \check{\psi}^{2}\right. \\
& +\tilde{C}_{2} \nabla_{\rho} \nabla_{\sigma} \check{\psi}^{2} \nabla^{\rho} \nabla^{\sigma} \check{\psi}^{2} \\
& \left.+\tilde{C}_{3} \nabla_{\rho} \nabla_{\sigma} \nabla_{\lambda} \check{\psi}^{2} \nabla^{\rho} \nabla^{\sigma} \nabla^{\lambda} \check{\psi}^{2}\right) \\
& +C_{1} \nabla_{\mu} \check{\psi}^{2} \nabla_{\nu} \check{\psi}^{2}+D_{1} \check{\psi}^{2} \nabla_{\mu} \nabla_{\nu} \check{\psi}^{2} \\
& +C_{2} \nabla_{\mu} \nabla^{\rho} \check{\psi}^{2} \nabla_{\nu} \nabla_{\rho} \check{\psi}^{2}+D_{2} \nabla^{\rho} \check{\psi}^{2} \nabla_{\mu} \nabla_{\nu} \nabla_{\rho} \check{\psi}^{2} \\
& +C_{3} \nabla_{\mu} \nabla^{\rho} \nabla^{\sigma} \check{\psi}^{2} \nabla_{\nu} \nabla_{\rho} \nabla_{\sigma} \check{\psi}^{2} \\
& +D_{3} \nabla^{\rho} \nabla^{\sigma} \check{\psi}^{2} \nabla_{\mu} \nabla_{\nu} \nabla_{\rho} \nabla_{\sigma} \check{\psi}^{2} .
\end{aligned}
$$

Then the equation of motion of the spin-two field $H_{\mu \nu}^{4}$ should read

$$
\begin{aligned}
& \left(\square-\frac{32}{L^{2}}\right) H_{\mu \nu}^{4}+g_{\mu \nu}\left(\alpha_{0} \Psi^{2} \Psi^{2}+\alpha_{1} \nabla_{\rho} \Psi^{2} \nabla^{\rho} \Psi^{2}\right) \\
& \quad+\beta_{1} \nabla_{\mu} \Psi^{2} \nabla_{\nu} \Psi^{2}+\beta_{2} \Psi^{2} \nabla_{\mu} \nabla_{\nu} \Psi^{2}=0 .
\end{aligned}
$$

Inserting (2.35) into (2.36) and comparing with (2.34), we determine the unknown coefficients as

$$
\begin{aligned}
& D_{3}=\frac{L^{6}}{1658880 \sqrt{10}}, \quad C_{3}=\frac{L^{6}}{1658880 \sqrt{10}}, \quad \tilde{C}_{3}=0, \\
& D_{2}=-\frac{L^{4}}{62208 \sqrt{10}}, \quad C_{2}=\frac{L^{4}}{124416 \sqrt{10}}, \\
& \tilde{C}_{2}=\frac{L^{4}}{165888 \sqrt{10}}, \quad D_{1}=\frac{103 L^{2}}{51840 \sqrt{10}}, \\
& C_{1}=\frac{113 L^{2}}{51840 \sqrt{10}}, \quad \tilde{C}_{1}=\frac{L^{2}}{7776 \sqrt{10}}, \\
& \tilde{C}_{0}=-\frac{7 \sqrt{10}+5184 \alpha_{1}}{10368}, \\
& \beta_{1}=\frac{1}{6 \sqrt{10}}, \quad \beta_{2}=\frac{1}{6 \sqrt{10}}, \quad \alpha_{0}=-\frac{4 \sqrt{10}+3240 \alpha_{1}}{135 L^{2}},
\end{aligned}
$$

and then write

$$
\begin{aligned}
& \left(\square-\frac{32}{L^{2}}\right) H_{\mu \nu}^{4} \\
& +g_{\mu \nu}\left(\frac{4 \sqrt{10}+3240 \alpha_{1}}{135 L^{2}} \Psi^{2} \Psi^{2}+\alpha_{1} \nabla_{\rho} \Psi^{2} \nabla^{\rho} \Psi^{2}\right) \\
& +\frac{1}{6 \sqrt{10}}\left(\nabla_{\mu} \Psi^{2} \nabla_{\nu} \Psi^{2}+\Psi^{2} \nabla_{\mu} \nabla_{\nu} \Psi^{2}\right)=0 .
\end{aligned}
$$

The asymptotic expansion of the LLM solution satisfies this equation up to quadratic order in the mass parameter, independent of the value of the constant $\alpha_{1}$. Since this constant plays no physical role, we can set it to zero and write

$$
\begin{aligned}
H_{\mu \nu}^{4}= & \check{h}_{\mu \nu}^{4}+\frac{1}{51840 \sqrt{10}} \\
& \times\left[-10 g_{\mu \nu}\left(35 \check{\psi}^{2} \check{\psi}^{2}-\frac{2 L^{2}}{3} \nabla_{\rho} \check{\psi}^{2} \nabla^{\rho}\right.\right. \\
& \left.\times \check{\psi}^{2}-\frac{L^{4}}{32} \nabla_{\rho} \nabla_{\sigma} \check{\psi}^{2} \nabla^{\rho} \nabla^{\sigma} \check{\psi}^{2}\right) \\
& +113 L^{2} \nabla_{\mu} \check{\psi}^{2} \nabla_{\nu} \check{\psi}^{2}+103 L^{2} \check{\psi}^{2} \nabla_{\mu} \nabla_{\nu} \check{\psi}^{2} \\
& +\frac{5 L^{4}}{12} \nabla_{\mu} \nabla^{\rho} \check{\psi}^{2} \nabla_{\nu} \nabla_{\rho} \check{\psi}^{2}-\frac{5 L^{4}}{6} \nabla^{\rho} \check{\psi}^{2} \nabla_{\mu} \nabla_{\nu} \nabla_{\rho} \check{\psi}^{2} \\
& +\frac{L^{6}}{32} \nabla_{\mu} \nabla^{\rho} \nabla^{\sigma} \check{\psi}^{2} \nabla_{\nu} \nabla_{\rho} \nabla_{\sigma} \check{\psi}^{2} \\
& \left.+\frac{L^{6}}{32} \nabla^{\rho} \nabla^{\sigma} \check{\psi}^{2} \nabla_{\mu} \nabla_{\nu} \nabla_{\rho} \nabla_{\sigma} \check{\psi}^{2}\right] .
\end{aligned}
$$

The equation of motion for the fourth traceless KK graviton mode is the traceless part of (2.38) and is given by

$$
\begin{aligned}
& \left(\square-\frac{32}{L^{2}}\right) H_{(\mu \nu)}^{4}+\frac{1}{6 \sqrt{10}} \\
& \left(\nabla_{(\mu} \Psi^{2} \nabla_{\nu)} \Psi^{2}+\Psi^{2} \nabla_{(\mu} \nabla_{\nu)} \Psi^{2}\right)=0,
\end{aligned}
$$


where

$$
\begin{aligned}
& H_{(\mu \nu)}^{4}=H_{\mu \nu}^{4}-\frac{1}{4} g_{\mu \nu} g^{\rho \sigma} H_{\rho \sigma}^{4} \\
& =\check{h}_{(\mu \nu)}^{4}+\frac{1}{1080 \sqrt{10}}\left[\frac { 1 } { 7 2 } g _ { \mu \nu } \left(303 \check{\psi}^{2} \check{\psi}^{2}-\frac{389 L^{2}}{8}\right.\right. \\
& \times \nabla_{\rho} \check{\psi}^{2} \nabla^{\rho} \breve{\psi}^{2}+\frac{5 L^{4}}{16} \nabla_{\rho} \nabla_{\sigma} \check{\psi}^{2} \nabla^{\rho} \nabla^{\sigma} \check{\psi}^{2} \\
& \left.-\frac{3 L^{6}}{256} \nabla_{\rho} \nabla_{\sigma} \nabla_{\lambda} \check{\psi}^{2} \nabla^{\rho} \nabla^{\sigma} \nabla^{\lambda} \breve{\psi}^{2}\right) \\
& +113 L^{2} \nabla_{\mu} \check{\psi}^{2} \nabla_{\nu} \check{\psi}^{2}+103 L^{2} \breve{\psi}^{2} \nabla_{\mu} \nabla_{\nu} \check{\psi}^{2} \\
& +\frac{5 L^{4}}{12} \nabla_{\mu} \nabla^{\rho} \breve{\psi}^{2} \nabla_{\nu} \nabla_{\rho} \check{\psi}^{2}-\frac{5 L^{4}}{6} \nabla^{\rho} \breve{\psi}^{2} \nabla_{\mu} \nabla_{\nu} \nabla_{\rho} \check{\psi}^{2} \\
& +\frac{L^{6}}{32} \nabla_{\mu} \nabla^{\rho} \nabla^{\sigma} \check{\psi}^{2} \nabla_{\nu} \nabla_{\rho} \nabla_{\sigma} \check{\psi}^{2} \\
& \left.+\frac{L^{6}}{32} \nabla^{\rho} \nabla^{\sigma} \breve{\psi}^{2} \nabla_{\mu} \nabla_{\nu} \nabla_{\rho} \nabla_{\sigma} \breve{\psi}^{2}\right] .
\end{aligned}
$$

The last equation is the KK map for the fourth KK graviton mode in quadratic order in the mass parameter. The nonlinear KK maps for the scalar and tensor modes given in (2.29) and (2.41), respectively, are crucial in holographic determination of the vevs of gauge invariant operators, which are dual to these KK modes. See Sect. 4.

\section{Gauge invariant operators and Vevs in mABJM theory}

In the previous section, we defined the physical modes in 4-dimensions using various non-linear KK maps including higher derivatives. These physical modes have corresponding operators by the gauge/gravity dictionary. In this section, we discuss possible operators with conformal dimension $\Delta=2$ in the ABJM theory and read the vevs of those operators in the large $N$ limit from the vacua of the mABJM theory.

\subsection{Vacua in the mABJM theory}

The mass term in the mABJM theory breaks the SU(4) global symmetry of the ABJM theory to $\mathrm{SU}(2) \times \mathrm{SU}(2) \times \mathrm{U}(1)$. According to the reduced global symmetry, we split the four-complex scalar fields in the ABJM theory as $Y^{A}=$ $\left(Z^{a}, W^{\dagger a}\right)$, where $A=1,2,3,4$ and $a, b=1,2$. Accordingly, the vacuum equation in the $\mathrm{mABJM}$ theory is written as

$$
\begin{aligned}
& Z^{a} Z_{b}^{\dagger} Z^{b}-Z^{b} Z_{b}^{\dagger} Z^{a}=-\frac{\mu k}{2 \pi} Z^{a}, \\
& W^{\dagger a} W_{b} W^{\dagger b}-W^{\dagger b} W_{b} W^{\dagger a}=\frac{\mu k}{2 \pi} W^{\dagger a},
\end{aligned}
$$

$W_{a} Z^{b} W_{b}-W_{b} Z^{b} W_{a}=0, \quad Z^{b} W_{b} Z^{a}-Z^{a} W_{b} Z^{b}=0$,

where $\mu$ is a mass parameter. The solutions of those vacuum equations have been obtained in [5] and are presented by a direct sums of two types of irreducible $n \times(n+1)$ matrices $\mathcal{M}_{a}^{(n)}(a=1,2)$ and their Hermitian conjugates, $\overline{\mathcal{M}}_{a}^{(n)}$. These rectangular matrices are referred as the GRVV matrices,



where $n=0,1, \ldots, N-1$. The vacuum solutions are given by





A given vacuum solution contains $N_{n}$ rectangular matrices of the type $\mathcal{M}_{a}^{(n)}$ and $N_{n}^{\prime}$ rectangular matrices of the type $\overline{\mathcal{M}}_{a}^{(n)}$. The set of parameters $\left\{N_{n}, N_{n}^{\prime}\right\}$ completely specifies a vacuum solution and they are called occupation numbers $[8$, 17]. Since $Z^{a}$ and $W^{\dagger a}$ are $N \times N$ matrices, the occupation numbers should satisfy the two constraints,

$$
N=\sum_{n=0}^{N-1}\left[\left(n+\frac{1}{2}\right)\left(N_{n}+N_{n}^{\prime}\right)\right], \quad \sum_{n=0}^{\infty} N_{n}=\sum_{n=0}^{\infty} N_{n}^{\prime} .
$$

At quantum level, some of vacuum solutions are not supersymmetric and only a subset of these classical solutions sat- 
isfying the conditions, $0 \leq N_{n}$ and $N_{n}^{\prime} \leq k$, remain to be supersymmetric [17].

\subsection{Gauge invariant operators in the ABJM theory}

In general, the CPOs of conformal dimension $\Delta$ in the mABJM theory are given by a trace of products of the four complex scalar fields $Y^{A}$ and their Hermitian conjugates $Y_{A}^{\dagger}$,

$\mathcal{O}^{(\Delta)}=C_{A_{1} \cdots A_{\Delta}}^{B_{1} \cdots B_{\Delta}} \operatorname{Tr}\left(Y^{A_{1}} Y_{B_{1}}^{\dagger} \cdots Y^{A_{\Delta}} Y_{B_{\Delta}}^{\dagger}\right)$.

These CPOs are dual to the KK scalar modes $\Psi^{I_{1}}$ with mass $M_{\Psi^{I_{1}}}^{2}=\frac{I_{1}\left(I_{1}-6\right)}{L^{2}}$ and conformal dimensions $\Delta=\frac{I_{1}}{2},\left\{I_{1}=\right.$ $2,4,6, \ldots\}[9,10]$. The dual gauge invariant operators for the other KK towers of scalar modes are the descendent of these CPOs, which are obtained by applying the supersymmetry generators of the $\mathcal{N}=6 \mathrm{mABJM}$ theory to $\mathcal{O}^{(\Delta)}$. In particular, the gauge invariant operators dual to the scalar modes $\Phi^{I_{1}}$ are obtained by applying six supersymmetry generators to the CPO and thus they are given by

$$
\begin{aligned}
\mathcal{O}_{6}^{(\Delta)}= & C_{a_{1} a_{2} a_{3} A_{1} \cdots A_{\Delta-6}}^{(6) b_{1} b_{2} b_{3} B_{1} \cdots B_{\Delta-6}} \operatorname{STr}\left(\psi^{\dagger a_{1}} \psi_{b_{1}} \psi^{\dagger a_{2}} \psi_{b_{2}} \psi^{\dagger a_{3}}\right. \\
& \left.\times \psi_{b_{3}} Y^{A_{1}} Y_{B_{1}}^{\dagger} \cdots Y^{A_{\Delta-6}} Y_{B_{\Delta-6}}^{\dagger}\right),
\end{aligned}
$$

where $\psi^{\dagger a}$ with $a=1,2,3,4$ are the four complex fermionic fields of the ABJM theory and STr denotes symmetrized trace. According to the relations between the mass of the scalar fields and the conformal dimension of the dual operators listed in $[9,10]$, the masses of the KK scalar modes $\Phi^{I_{1}}$ are $M_{\Phi^{I_{1}}}^{2}=\frac{\left(I_{1}+12\right)\left(I_{1}+6\right)}{L^{2}}$ and their conformal dimensions are $\Delta=\frac{I_{1}+12}{2},\left\{I_{1}=0,2,4, \ldots\right\}$. Therefore, the gauge invariant operator dual to the scalar mode $\Phi^{4}$ is

$$
\begin{aligned}
\mathcal{O}_{6}^{(\Delta=8)}= & C_{a_{1} a_{2} a_{3} a_{1} A_{2}}^{(6) b_{1} b_{2} B_{2}} \operatorname{STr}\left(\psi^{\dagger a_{1}} \psi_{b_{1}} \psi^{\dagger a_{2}} \psi_{b_{2}}\right. \\
& \left.\times \psi^{\dagger a_{3}} \psi_{b_{3}} Y^{A_{1}} Y_{B_{1}}^{\dagger} Y^{A_{2}} Y_{B_{2}}^{\dagger}\right),
\end{aligned}
$$

whereas the scalar field $\Psi^{4}$ is dual to the CPO,

$\mathcal{O}^{(\Delta=2)}=C_{A_{1} A_{2}}^{B_{1} B_{2}} \operatorname{Tr}\left(Y^{A_{1}} Y_{B_{1}}^{\dagger} Y^{A_{2}} Y_{B_{2}}^{\dagger}\right)$.

In our previous paper, we defined the CPO with $\Delta=1$, which reflects the global $\mathrm{SU}(2) \times \mathrm{SU}(2) \times \mathrm{U}(1)$ symmetry of the $\mathrm{mABJM}$ theory. The form of the CPO is given by

$\mathcal{O}^{(\Delta=1)}=\mathcal{N}_{1} \operatorname{Tr}\left(Y^{1} Y_{1}^{\dagger}+Y^{2} Y_{2}^{\dagger}-Y^{3} Y_{3}^{\dagger}-Y^{4} Y_{4}^{\dagger}\right)$,

where $\mathcal{N}_{1}$ is the normalization factor. The procedure to determine the form of the $\mathcal{O}^{(\Delta=1)}$ was explained in the Appendix A.4 of $[9,10]$. However, we fix the normalization factor $\mathcal{N}_{1}$ in a different way, which matches the GKP-W relation $[2,3]$ in the gauge/gravity dictionary. We will explain the details later.

In this section, we consider the $\mathrm{CPO}$ with $\Delta=2$, which reflects the global $\mathrm{SU}(2) \times \mathrm{SU}(2) \times \mathrm{U}(1)$ symmetry of the $\mathrm{mABJM}$ theory. Using a similar procedure as in the Appendix A. 4 of $[9,10]$, we determine the relations among the constants $C_{A_{1} A_{2}}^{B_{1} B_{2}}$ in (3.8) and construct the CPO with $\Delta=2$ with the global $\mathrm{SU}(2) \times \mathrm{SU}(2) \times \mathrm{U}(1)$ symmetry as ${ }^{4}$

$$
\begin{aligned}
\mathcal{O}^{(\Delta=2)}= & \mathcal{N}_{2}\left[\sum_{A, B=1}^{2} \operatorname{Tr}\left(Y^{A} Y_{A}^{\dagger} Y^{B} Y_{B}^{\dagger}\right)\right. \\
& +\sum_{A, B=1}^{2} \operatorname{Tr}\left(Y^{A} Y_{B}^{\dagger} Y^{B} Y_{A}^{\dagger}\right) \\
& +\sum_{A, B=3}^{4} \operatorname{Tr}\left(Y^{A} Y_{A}^{\dagger} Y^{B} Y_{B}^{\dagger}\right)+\sum_{A, B=3}^{4} \operatorname{Tr}\left(Y^{A} Y_{B}^{\dagger} Y^{B} Y_{A}^{\dagger}\right) \\
& -3 \sum_{A=1}^{2} \sum_{B=3}^{4} \operatorname{Tr}\left(Y^{A} Y_{A}^{\dagger} Y^{B} Y_{B}^{\dagger}\right) \\
& \left.-3 \sum_{A=1}^{2} \sum_{B=3}^{4} \operatorname{Tr}\left(Y^{A} Y_{B}^{\dagger} Y^{B} Y_{A}^{\dagger}\right)\right]
\end{aligned}
$$

where $\mathcal{N}_{2}$ is the normalization factor. We will fix the normalization factor later by use of the GKP-W relation.

In order to obtain the vevs of the above CPOs, we expand the complex scalar fields near the vacuum as

$Y^{A}=Y_{0}^{A}+\hat{Y}^{A}$

where $Y_{0}^{A}$ 's denote the discrete Higgs vacua discussed above and $\hat{Y}^{A}$ 's are the complex scalar operators representing fluctuations around the vacua. Then the vev of a CPO in the mABJM theory is given by $[9,10]$

$\left\langle\mathcal{O}^{(\Delta)}\right\rangle_{m}=\left.\mathcal{O}^{(\Delta)}\right|_{Y^{A}=Y_{0}^{A}}+\sum_{i}\left\langle\delta \mathcal{O}_{i}^{(\Delta)}\right\rangle_{0}+\mathcal{O}\left(\frac{1}{N}\right)$

where $\langle\cdots\rangle_{m}$ and $\langle\cdots\rangle_{0}$ denote the vevs of an operator in the mABJM theory and the ABJM theory, respectively, and $\delta \mathcal{O}_{i}^{(\Delta)}$ is an operator containing at least one $\hat{Y}^{A}$ or $\hat{Y}^{\dagger A}$. The $\frac{1}{N}$-corrections come from the contributions of multi-trace terms. The second term is a one point function in a conformally symmetric ABJM theory and is vanishing. Therefore, in the large $N$ limit, we have

$\left\langle\mathcal{O}^{(\Delta)}\right\rangle_{m}=\left.\mathcal{O}^{(\Delta)}\right|_{Y^{A}=Y_{0}^{A}}$

\footnotetext{
${ }^{4}$ See also the Appendix of the current paper for the details.
} 
We will display the explicit forms of the vevs for CPOs of conformal dimensions $\Delta=1$ and $\Delta=2$ in Sect. 5 .

\section{Asymptotic behavior of LLM geometries and 4-dimensional KK modes}

The metric for the LLM geometries with $\mathbb{Z}_{k}$ orbifold, which have $\mathrm{SO}(2,1) \times \mathrm{SO}(4) / \mathbb{Z}_{k} \times \mathrm{SO}(4) / \mathbb{Z}_{k}$ isometry $[8,18]$, is given by

$$
\begin{aligned}
d s^{2}= & \frac{L^{2}}{4 z^{2}}\left[d z^{2}+\frac{4 z^{2}}{L^{2}}\left(1+\tilde{g}_{1}(z, \tau)\right)\left(-d t^{2}+d w_{1}^{2}+d w_{2}^{2}\right)\right] \\
& +\frac{L^{2}}{4\left(1-\tau^{2}\right)}\left(1+\tilde{g}_{2}(z, \tau)\right) d \tau^{2} \\
& +\frac{L^{2}(1+\tau)}{2}\left(1+\tilde{g}_{3}(z, \tau)\right) d s_{S^{3} / \mathbb{Z}_{k}}^{2} \\
& +\frac{L^{2}(1-\tau)}{2}\left(1+\tilde{g}_{4}(z, \tau)\right) d s_{\tilde{S}^{3} / \mathbb{Z}_{k}}^{2}
\end{aligned}
$$

where the $\tilde{g}_{i}(z, \tau)$ represent the deviation of the LLM metric from the $\mathrm{AdS}_{4} \times S^{7}$ background. See [9,10] for details. Similarly, the 4-form field strength of the LLM geometries can be split into the background and the fluctuations. The values of the various KK modes $\left(h_{\mu \nu}^{I_{1}}, \phi^{I_{1}}, \ldots\right)$, introduced in Sect. 2, are read from the asymptotic expansion of $\tilde{g}_{i}(z, \tau)$ and the similar functions in 4-form field strength. In $[9,10]$, we have listed the full result for all the KK modes up to $\mu_{0}^{2}$ order. As mentioned in the previous section, here we focus on the equations of motion for the fourth KK scalar and graviton modes. For the quadratic parts in the equations of motion and in the KK maps discussed in the previous section, we also need the asymptotic expansion of $\breve{\psi}^{2}$. Then we take the following results for the 11-dimensional modes from $[9,10]$

$$
\begin{aligned}
\check{h}_{i j}^{4}= & {\left[-\frac{3 L^{2} \mu_{0}^{2}}{4 \sqrt{10}} \beta_{3}^{2}+\mathcal{O}\left(\mu_{0}^{4}\right)\right] \eta_{i j}, } \\
\check{h}_{z z}^{4}= & -\frac{L^{2} \mu_{0}^{2}}{4 \sqrt{10}} \beta_{3}^{2}+\mathcal{O}\left(\mu_{0}^{4}\right), \\
\check{\psi}^{4}= & -\frac{2 \sqrt{10}\left(\mu_{0} z\right)^{2}}{35}\left(3780 \beta_{2}^{3}+758 \beta_{3}^{2}-945 \beta_{2} \beta_{4}\right) \\
& +\mathcal{O}\left(\mu_{0}^{4}\right), \\
\check{\phi}^{4}= & -\frac{44 \sqrt{10}\left(\mu_{0} z\right)^{2}}{7} \beta_{3}^{2}+\mathcal{O}\left(\mu_{0}^{4}\right), \\
\check{\psi}^{2}= & -24 \beta_{3} \mu_{0} z+\mathcal{O}\left(\mu_{0}^{3}\right),
\end{aligned}
$$

where $\eta_{i j}=\operatorname{diag}(-1,1,1)$ and

$$
\begin{aligned}
& \beta_{2}=C_{2}-C_{1}^{2}, \quad \beta_{3}=C_{3}-3 C_{1} C_{2}+2 C_{1}^{3}, \\
& \beta_{4}=C_{4}+3 C_{2}^{2}-4 C_{1} C_{3} .
\end{aligned}
$$

The parameters $C_{p}$ were introduced in [19-21],

$$
C_{p}=\sum_{i=1}^{\infty}(-1)^{i+1}\left(\frac{\tilde{x}_{i}}{2 \pi l_{\mathrm{P}}^{3} \mu_{0} \sqrt{A}}\right)^{p},
$$

where $A$ is defined by

$$
A=k N-\frac{1}{2} \sum_{n=0}^{\infty}\left[l_{n}\left(k-l_{n}\right)+l_{n}^{\prime}\left(k-l_{n}^{\prime}\right)\right]
$$

with the discrete torsions $\left(l_{n}, l_{n}^{\prime}\right)$ introduced in [8]. In the Young diagram representation of the LLM solutions, $A$ means the area of the Young diagram $[9,10]$.

In the previous section, we have established the KK maps which relate the above 11-dimensional KK modes to the corresponding canonical 4-dimensional gravity fields. These maps are given in (2.29) and (2.41). These maps express the asymptotic expansions of the fourth KK scalar and graviton modes as follows

$$
\begin{aligned}
\Phi^{4} & =\mathcal{O}\left(\left(\mu_{0} z\right)^{4}\right), \\
\Psi^{4} & =-54 \sqrt{10}\left(4 \beta_{2}^{3}+\beta_{3}^{2}-\beta_{2} \beta_{4}\right)\left(\mu_{0} z\right)^{2}+\mathcal{O}\left(\left(\mu_{0} z\right)^{4}\right) \\
H_{(i j)}^{4} & =\frac{L^{2}}{4 z^{2}}\left[-\frac{4\left(\mu_{0} z\right)^{2}}{\sqrt{10}} \beta_{3}^{2}+\mathcal{O}\left(\left(z \mu_{0}\right)^{4}\right)\right] \eta_{i j} \\
H_{(z z)}^{4} & =\frac{L^{2}}{4 z^{2}}\left[\frac{12\left(\mu_{0} z\right)^{2}}{\sqrt{10}} \beta_{3}^{2}+\mathcal{O}\left(\left(\mu_{0} z\right)^{4}\right)\right] .
\end{aligned}
$$

For clarity of presentation, we also rewrite the similar results for the zeroth and second KK graviton modes obtained in [16] and $[9,10]$, respectively,

$$
\begin{aligned}
& H_{i j}^{0}=\frac{L^{2}}{4 z^{2}}\left[-\frac{\left(\mu_{0} z\right)^{2}}{45}\left(30+\beta_{3}^{2}\right)+\mathcal{O}\left(\left(\mu_{0} z\right)^{4}\right)\right] \eta_{i j} \\
& H_{z z}^{0}=\frac{L^{2}}{4 z^{2}}\left[-\frac{\left(\mu_{0} z\right)^{2}}{360}\left(960+29 \beta_{3}^{2}\right)+\mathcal{O}\left(\left(\mu_{0} z\right)^{4}\right)\right], \\
& H_{\mu \nu}^{2}=\frac{L^{2}}{4 z^{2}}\left[0+\mathcal{O}\left(\left(\mu_{0} z\right)^{3}\right)\right] .
\end{aligned}
$$

The Fefferman-Graham (FG) coordinate system is more convenient for the implementation of the gauge/gravity dictionary. Therefore, we write the asymptotically $\mathrm{AdS}_{4} 4$ dimensional metric $\left(\hat{g}_{\mu \nu}=g_{\mu \nu}^{A d S_{4}}+H_{\mu \nu}^{0}\right)$ in the FG coordinate by using the coordinate transformation $z=\tilde{z}+$ $\frac{\mu_{0}^{2}\left(960+29 \beta_{3}^{2}\right)}{1440} \tilde{z}^{3}$,

$$
\begin{aligned}
d s^{2}= & \frac{L^{2}}{4 \tilde{z}^{2}}\left[d \tilde{z}^{2}+\left(1-\left(2+\frac{\beta_{3}^{2}}{16}\right)\left(\mu_{0} \tilde{z}\right)^{2}\right.\right. \\
& \left.\left.+\mathcal{O}\left(\left(\mu_{0} \tilde{z}\right)^{4}\right)\right) \eta_{i j} d x^{i} d x^{j}\right] .
\end{aligned}
$$


Since all the terms in (4.6) are already at least quadratic in $\mu_{0}$, the above coordinate transformation only amounts to replacing $z$ by $\tilde{z}$ in those terms.

As mentioned in the previous section, the scalar field $\Psi^{4}$ with $M_{\Psi^{4}}^{2}=\left.\frac{I(I-6)}{L^{2}}\right|_{I=4}$ is dual to a CPO of conformal dimension $\Delta=\left.\frac{I}{2}\right|_{I=4}=2$ while the scalar field $\Phi^{4}$ with $M_{\Phi^{4}}^{2}=$ $\left.\frac{(I+12)(I+6)}{L^{2}}\right|_{I=4}$ is dual to a gauge invariant operator with conformal dimension $\Delta=\left.\frac{I+12}{2}\right|_{I=4}=8$. The GKP-W relation states that the vev of a $\mathrm{CPO}\left(\mathcal{O}^{\Delta}\right)$ of conformal dimension $\Delta$ is determined by the coefficient of $z^{\Delta}$ in the asymptotic expansion of the dual scalar field. Thus the vev of the CPO in terms of the holographic renormalizaton [22-31] is given by

$$
\left\langle\mathcal{O}^{\Delta=2}\right\rangle_{\mathrm{HR}}=-54 \mathbb{N} \sqrt{10} \mu_{0}^{2}\left(4 \beta_{2}^{3}+\beta_{3}^{2}-\beta_{2} \beta_{4}\right),
$$

where $\mathbb{N}$ is some normalization factor to be fixed later.

Similarly, the gauge/gravity dictionary maps the metric to the stress-energy tensor $T_{i j}$ of the dual gauge theory. Writing the $(d+1)$-dimensional metric in the FG coordinate

$d s^{2}=\frac{L_{\text {AdS }}^{2}}{\tilde{z}^{2}}\left[d \tilde{z}^{2}+g_{i j}(x, \tilde{z}) d x^{i} d x^{j}\right]$

with the asymptotic expansion of the function $g_{i j}(x, \tilde{z})$ given by

$g_{i j}(x, \tilde{z})=g_{(0) i j}(x)+\tilde{z}^{2} g_{(2) i j}(x)+\cdots+\tilde{z}^{d} g_{(d) i j}(x)+\cdots$,

then the vev of the stress-energy tensor is given by [22-25]

$\left\langle T_{i j}\right\rangle_{\mathrm{HR}}=\frac{d L_{\mathrm{AdS}}^{d-1}}{16 \pi G_{N}} g_{(d) i j}$.

From (4.8) we read that the asymptotic expansion does not contain the $\tilde{z}^{3}$ term with $d=3$ in (4.12), which implies that the vev of the stress-energy tensor of the mABJM theory is vanishing as required by the supersymmetry of the theory.

The non-zero KK graviton modes $H_{\mu \nu}^{2}$ and $H_{\mu \nu}^{4}$ are dual to the operators

$T_{i j}^{(2)}=C_{A}^{B} \operatorname{STr}\left(T_{i j} Y^{A} Y_{B}^{\dagger}\right)$,

$T_{i j}^{(4)}=C_{A A^{\prime}}^{B B^{\prime}} \operatorname{STr}\left(T_{i j} Y^{A} Y_{B}^{\dagger} Y^{A^{\prime}} Y_{B^{\prime}}^{\dagger}\right)$,

respectively. The vevs of these operators are given by

$\left\langle T_{i j}^{(2)}\right\rangle_{\mathrm{HR}}=\mathbb{N}_{2} g_{(d-1) i j}^{(2)}, \quad\left\langle T_{i j}^{(4)}\right\rangle_{\mathrm{HR}}=\mathbb{N}_{4} g_{(d) i j}^{(4)}$,

where $g_{(d-1) i j}^{(2)}$ is the coefficient of $\tilde{z}^{d-1=2}$ in the expansion of $H_{i j}^{2}$ and $g_{(d) i j}^{(4)}$ is the coefficient of $\tilde{z}^{d=3}$ in the expansion of $H_{i j}^{4}$. From (4.6) and (4.7), we see that the expansion of $H_{i j}^{2}$ contains only odd powers of $\tilde{z}$ whereas the expansion of $H_{i j}^{4}$ contains only even powers of $\tilde{z}$. Therefore, the vevs of both $T_{i j}^{(2)}$ and $T_{i j}^{(4)}$ are vanishing.

\section{Vevs of CPOs and GKP-W relation}

In our previous work [16], we have constructed the 4-dimensional gravity action with two scalar fields, $T$ and $\Psi_{(1)}$, after the KK reduction from the 11-dimensional supergravity. The field $T$ is dual to a gauge invariant operator, $\tilde{\mathcal{O}}^{(2)}=\tilde{C}_{A}^{B} \operatorname{Tr}\left(\psi^{\dagger A} \psi_{B}\right)$ with $\Delta=2$ and the field $\Psi_{(1)}$ is dual to the CPO (3.9).

In this section, we focus on the GKP-W relation for the CPOs with $\Delta=1,2$. For that purpose, we consider the 4-dimensional gravity action with two scalar fields, $\Psi_{(1)}$ and $\Psi_{(2)}$,

$$
\begin{aligned}
S= & \frac{1}{16 \pi G_{4}} \int d^{4} x \sqrt{-g}(\hat{R}-2 \Lambda) \\
& -\sum_{i=1}^{2}\left[\frac{A \Psi_{(i)}}{2} \int d^{4} x \sqrt{-g}\left(\partial_{\mu} \Psi_{(i)} \partial^{\mu} \Psi_{(i)}+M_{\Psi_{(i)}}^{2} \Psi_{(i)}^{2}\right)\right] \\
= & \frac{N^{2}}{3 \sqrt{2 \pi^{2} \lambda} L^{2}} \int d^{4} x \sqrt{-g} \\
& \times\left[\hat{R}-2 \Lambda-\frac{1}{2} \sum_{i=1}^{2}\left(\partial_{\mu} \tilde{\Psi}_{(i)} \partial^{\mu} \tilde{\Psi}_{(i)}+M_{\tilde{\Psi}_{(i)}}^{2} \tilde{\Psi}_{(i)}^{2}\right)\right],
\end{aligned}
$$

where

$\frac{1}{16 \pi G_{4}}=\frac{N^{2}}{3 \sqrt{2 \pi^{2} \lambda} L^{2}}$

with the 't Hooft coupling $\lambda=N / k$ in the ABJM theory [6]. In order to obtain the normalization which is consistent with the GKP-W relation in the literature, we rescaled the scalar fields as

$\tilde{\Psi}_{(i)}=\sqrt{16 \pi G_{4} A \Psi_{(i)}} \Psi_{(i)}$.

Solutions for the rescaled fields are read from the asymptotic expansion of the LLM geometries,

$\tilde{\Psi}_{(1)}=-\frac{1}{\sqrt{2}} \beta_{3} \mu_{0} z+\mathcal{O}\left(\mu_{0}^{3}\right)$,

$\tilde{\Psi}_{(2)}=-\frac{1}{\sqrt{2}}\left(4 \beta_{2}^{3}+\beta_{3}^{2}-\beta_{2} \beta_{4}\right)\left(\mu_{0} z\right)^{2}+\mathcal{O}\left(\mu_{0}^{4}\right)$,

where we set the scaling factor in (5.3) as $\sqrt{16 \pi G_{4} A_{\Psi_{(1)}}}=$ $\frac{1}{24 \sqrt{2}}$ by reading the value of $A_{\Psi_{(1)}}$ from the equation of motion of $H_{\mu \nu}^{0}$ at $\mu_{0}^{2}$ order obtained in [16]. However, the scaling factor $\sqrt{16 \pi G_{4} A_{\Psi_{(2)}}}$ in (5.3) cannot be fixed without the information for the equation of motion of $H_{\mu \nu}^{0}$ at $\mu_{0}^{4}$-order. Since we do not have the equation of motion of $H_{\mu \nu}^{0}$ up to $\mu_{0}^{4}$-order, we choose this scaling factor as $\sqrt{16 \pi G_{4} A \Psi_{(2)}}=\frac{1}{108 \sqrt{5}}$ for later convenience.

As we mentioned in Sect. 4, the GKP-W relation imply, for odd dimensional QFT, the vev of a gauge invariant operator with conformal dimension $\Delta$ is obtained from the holographic renormalization procedure [22-31] in the large $N$ limit, 
Fig. 1 a Symmetric droplet representation of the LLM geometry, where the number of black strip is one, the length of it is $a$, and $k=\frac{a}{n}$ with integer $n$. b Young diagram corresponding to the droplet picture (a)

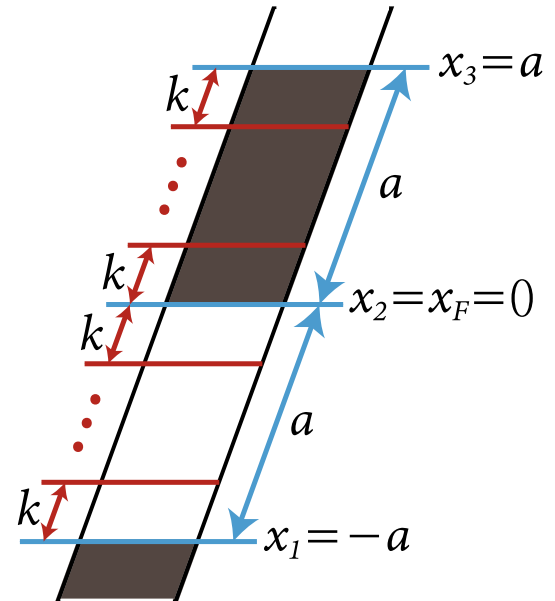

(a)

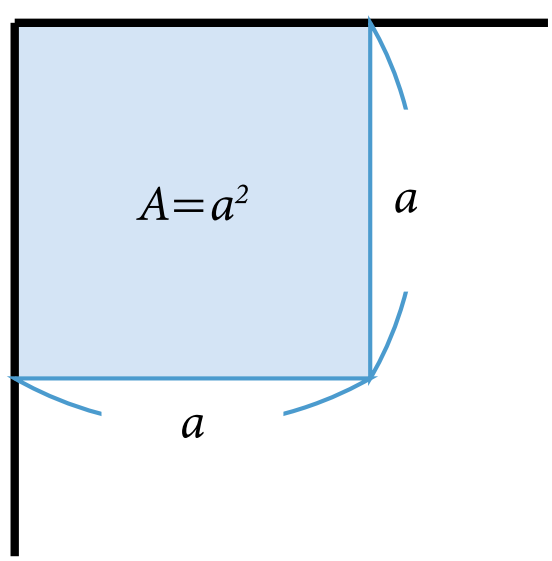

(b)
$\left\langle\mathcal{O}^{(\Delta)}\right\rangle_{\mathrm{HR}}=\frac{N^{2}}{3 \sqrt{2 \pi^{2} \lambda}}(2 \Delta-d) \tilde{\psi}_{\Delta}^{(i)}$

where $\tilde{\psi}_{\Delta_{\tilde{\Psi}}}^{(i)}$ is the coefficient of $z^{\Delta}$ in the asymptotic expansion of the field $\tilde{\Psi}_{(i)}$. Inserting the solutions (5.4) into (5.5), we obtain

$$
\begin{aligned}
\left\langle\mathcal{O}^{(\Delta=1)}\right\rangle_{\mathrm{HR}} & =-\frac{N^{2}}{3 \sqrt{2 \pi^{2} \lambda}} \tilde{\psi}_{1}^{(1)}=\frac{N^{2} \beta_{3} \mu_{0}}{6 \pi \sqrt{\lambda}}, \\
\left\langle\mathcal{O}^{(\Delta=2)}\right\rangle_{\mathrm{HR}} & =\frac{N^{2}}{3 \sqrt{2 \pi^{2} \lambda}} \tilde{\psi}_{2}^{(2)}=-\frac{N^{2}}{6 \pi \sqrt{\lambda}}\left(4 \beta_{2}^{3}+\beta_{3}^{2}-\beta_{2} \beta_{4}\right) \mu_{0}^{2} .
\end{aligned}
$$

The normalization factors $\mathcal{N}_{1,2}$ of the CPOs defined in (3.9) and (3.10) are determined from (5.6). ${ }^{5}$ For the CPO of conformal dimension $\Delta=1$, the vev (3.13) of the mABJM theory in the large $N$ limit can be read as $[9,10]$

$$
\begin{aligned}
\left\langle\mathcal{O}^{(\Delta=1)}\right\rangle_{m} & =\left.\mathcal{N}_{1} \operatorname{Tr}\left(Y^{1} Y_{1}^{\dagger}+Y^{2} Y_{2}^{\dagger}-Y^{3} Y_{3}^{\dagger}-Y^{4} Y_{4}^{\dagger}\right)\right|_{Y^{A}=Y_{0}^{A}} \\
& =\frac{2 \mathcal{N}_{1} N^{2} \beta_{3} \mu_{0}}{3 \pi \sqrt{\lambda}}
\end{aligned}
$$

where $\langle\cdots\rangle_{m}$ represents the vev of an operator in the mABJM theory. Comparing the vev in terms of the holographic renormalization in (5.6) with that of the mABJM theory in (5.7), we fix the normalization factor of $\mathcal{O}^{(1)}$ as $\mathcal{N}_{1}=\frac{1}{4}$. Thus the definition

\footnotetext{
$\overline{5 \text { As we see in }}$ (5.1), we used the overall normalization $1 /\left(16 \pi G_{4}\right)$ defined in (5.2) for the scalar field action. Due this normalizaton, one can drive the GKP-W relation in (5.5). However, there exists still an ambiguity in the definition of the one-point function, which comes from the definition of the source term in the generating functional of the $n$ point functions. In order to fix this ambiguity denoted as $\mathcal{N}_{1,2}$ in our case, one has to compare the values of the one point functions in the field theory and those in the supergravity. For the detailed discussion for this issue, for instance, see (5.19)-(5.21) in [11], where the normalization factors were fixed from the values of two point correlation functions.
}

of $\mathcal{O}^{(1)}$ in this paper has a factor of $\frac{1}{\sqrt{2}}$ difference from that of the previous paper $[9,10,16]$.

In order to fix the normalization factor $\mathcal{N}_{2}$ in (3.10), we consider a symmetric droplet case with $k \neq 1$. The corresponding droplet and Young diagram representations in the LLM geometries are depicted in Fig.1. In this case, we set $k=\frac{a}{n}, N=n a$, and $A=k N=a^{2}$. Then by fixing the coordinate of the Fermi level as $\tilde{x}_{2}=\tilde{x}_{F}=0,{ }^{6}$ we obtain

$C_{1}=C_{3}=0, \quad C_{2}=C_{4}=2$.

Using these values in the second line of (5.6), we obtain

$\left\langle\mathcal{O}^{(\Delta=2)}\right\rangle_{\mathrm{HR}}=-\frac{2 N^{2}}{3 \pi \sqrt{\lambda}} \mu_{0}^{2}$.

Now we try to calculate the corresponding vev in the field theory side. For the symmetric droplet case, one can also assign the discrete torsions as

$$
\begin{aligned}
& \left(l_{0}, l_{1}, \cdots, l_{n-1}\right)=\left(\frac{a}{n}, \frac{a}{n}, \cdots, \frac{a}{n}\right), \\
& \left(l_{0}^{\prime}, l_{1}^{\prime}, \cdots, l_{n-1}^{\prime}\right)=\left(\frac{a}{n}, \frac{a}{n}, \cdots, \frac{a}{n}\right) .
\end{aligned}
$$

Other values of discrete torsions are vanishing. Identifying the discrete torsions $\left\{l_{n}, l_{n}^{\prime}\right\}$ with the occupation numbers of GRVV matrices $\left\{N_{n}, N_{n}^{\prime}\right\}$, we calculate the vev of $\mathcal{O}^{(\Delta=2)}$ in (3.10) in the large $N$ limit,

$\left\langle\mathcal{O}^{(\Delta=2)}\right\rangle_{m}=\frac{2 k \mu_{0}^{2} \mathcal{N}_{2} N^{2}}{\pi^{2}}+\mathcal{O}(N)$,

where we have used the relations

\footnotetext{
${ }^{6}$ For the details of the droplet and Young diagram representations in the LLM geometries, see [8-10].
} 




Fig. 2 Validity of the holographic renormalization for the CPO of conformal dimension $\Delta=2$ in the square-shaped Young diagram of the LLM geometries at large $N$. The vertical axis is $K(N)=\frac{\left\langle\mathcal{O}^{(\Delta=2)}\right\rangle_{m}}{\left\langle\mathcal{O}^{(\Delta=2)}\right\rangle_{\mathrm{HR}}}$ with $N=4,9,16, \cdots, 225$

$$
\begin{aligned}
& \left.\operatorname{Tr}\left(\sum_{A=1}^{4} Y^{A} Y_{A}^{\dagger} Y^{A} Y_{A}^{\dagger}\right)\right|_{Y^{A}=Y_{0}^{A}}=\frac{4 k \mu_{0}^{2} N^{2}}{3 \pi^{2}}+\mathcal{O}(N), \\
& \operatorname{Tr}\left(Y^{1} Y_{1}^{\dagger} Y^{2} Y_{2}^{\dagger}+Y_{1}^{\dagger} Y^{1} Y_{2}^{\dagger} Y^{2}+Y^{3} Y_{3}^{\dagger} Y^{4} Y_{4}^{\dagger}\right. \\
& \left.\quad+Y_{3}^{\dagger} Y^{3} Y_{4}^{\dagger} Y^{4}\right)\left.\right|_{Y^{A}=Y_{0}^{A}}=\frac{2 k \mu_{0}^{2} N^{2}}{3 \pi^{2}}+\mathcal{O}(N) .
\end{aligned}
$$

Other combinations of the traces in (3.10) are vanishing due to the gauge choice of the vacuum solutions in [5]. Comparing the vev in the field theory side with that in gravity theory side, we fix the normalization factor in (3.10) as

$\mathcal{N}_{2}=-\frac{\pi}{3 \sqrt{k N}}$

We examine validity of the holographic renormalization (5.9) at large $N$ in Fig. 2. We expect that this normalization factor can be used in the calculation of $n$-point correlation functions.

In general the normalization factors of operators in the gauge/gravity duality are fixed in terms of two point functions in conformal field theory, by comparing the quantities in field theory side and the corresponding ones in gravity side [11]. However, in our case the forms of operators (for instance, see (3.10)) are too complicated, and so, it is a non-trivial problem to calculate the corresponding two point function in gravity side. It will be interesting if one can calculate the two point function for $\mathcal{O}^{(\Delta=2)}$ in gravity side in the future and check the validity of the normalization factor in (5.13), which was fixed by the calculation of the one-point function.

\section{Conclusion}

In this paper, we obtained the vevs of gauge invariant operators up to $\mu_{0}^{2}$-order in terms of the holographic renormaliza- tion in the mABJM theory. We found that the vevs of gauge invariant operators are vanishing up to $\mu_{0}^{2}$-order expect for the case of the CPOs with conformal dimension $\Delta=1,2$. For the latter cases, the vevs were obtained using the KK holography in the large $N$ limit. In order to show validity of the holographic relation, we compared the vevs from the supersymmetric vacua of the mABJM theory with those from the LLM solutions. Our results for the CPO of conformal dimension $\Delta=2$ are limited to the cases of the LLM solutions, which are represented by a square-shaped Young diagrams. We showed that the vevs obtained from the mABJM theory with an appropriate normalization of the CPO of conformal dimension $\Delta=2$ approach those obtained from the holographic renormalization at large $N$.

The result we obtained in this paper is a further confirmation of the claim in $[9,10]$ about duality between the mABJM theory and the 11-dimensional supergravity on the LLM geometry. However, in the present case the procedure is highly non-trivial. In order to read the vevs of the CPO of conformal dimension $\Delta=2$ from the asymptotic expansion of the LLM solutions, we need to carry out the KK reduction of the 11-dimensional supergravity and then construct a 4-dimensional gravity on the asymptotic $\mathrm{AdS}_{4}$ background. Unlike the case of the CPO of conformal dimension $\Delta=1$, we need to establish the KK maps in the quadratic order between the 4-dimensional fields and the 11dimensional fields. The KK maps include the non-trivial field redefinitions, which are required to absorb higher derivative terms and result in the canonical equations of motion for the 4-dimensional fields. Identifying the 4-dimensional fields obtained from the KK maps with the fluctuations obtained from the asymptotic expansion of the LLM solutions, we read the asymptotically $\mathrm{AdS}_{4}$ solutions in the 4-dimensional equations of motion. We read the vevs of the CPO of conformal dimension $\Delta=2$ from those asymptotic solutions in 4-dimensions. We also confirm that the vevs of other gauge invariant operators which are not CPO as well as those of the massive KK graviton modes are vanishing.

In the previous work $[9,10]$, we showed that the vevs of $\mathcal{O}^{(\Delta=1)}$ for any LLM solutions in the holographic renormalization method are exactly the same as those of the mABJM theory in the large $N$ limit, i.e., $\left\langle\mathcal{O}^{(\Delta=1)}\right\rangle_{\mathrm{HR}}=\left\langle\mathcal{O}^{(\Delta=1)}\right\rangle_{m}$. This result heavily depends on the fact that the curvature in the asymptotic limit $\left(\mu_{0} z \ll 1\right)$ becomes weak for any LLM solutions [32]. Since the vev $\left\langle\mathcal{O}^{(\Delta=1)}\right\rangle_{\mathrm{HR}}$ is completely determined by the asymptotic expansion of the LLM solutions in $\mu_{0}$-order $[9,10]$, one can expect that the relation $\left\langle\mathcal{O}^{(\Delta=1)}\right\rangle_{\mathrm{HR}}=\left\langle\mathcal{O}^{(\Delta=1)}\right\rangle_{m}$ in the large $N$ limit is satisfied for all LLM solutions. However, by increasing the $\mu_{0} z$-value in the LLM geometry, we notice that some LLM geometries, which include short edges in the Young diagram representation, become strongly curved even in the large $N$ limit [32]. Therefore, in order to obtain the correct holographic rela- 
tion $(\Delta \neq 1$ ) for LLM geometries including strongly curved regions, one needs quantum corrections from the gravity side in the large $N$ limit,

$\left\langle\mathcal{O}^{(\Delta)}\right\rangle_{m}=\left\langle\mathcal{O}^{(\Delta)}\right\rangle_{\mathrm{HR}}+$ quantum corrections.

In other words, the LLM geometries with square-shaped Young diagrams do not include any short edges in the large $N$ limit and thus these geometries are weakly curved over all transverse regions. For these LLM geometries, we expect that the holographic relation (6.1) is satisfied without quantum corrections in the gravity side. In this paper, we examined validity of the vevs of $\mathcal{O}^{(\Delta=2)}$ in the holographic renormalization for the square-shaped Young-diagrams in the LLM geometries, and showed that $\left\langle\mathcal{O}^{(\Delta=2)}\right\rangle_{\mathrm{HR}}$ is approaching the value of $\left\langle\mathcal{O}^{(\Delta=2)}\right\rangle_{m}$ in the field theory side by increasing $N$. This result matches our expectation. It is also intriguing to examine the relation (6.1) for other Young diagrams in the LLM geometries.

Acknowledgements OK appreciates APCTP for its hospitality during completion of this work and DT would like to thank the physics department of Addis Ababa University for hospitality, during the visit to present part of this work. This work was supported by the National Research Foundation of Korea(NRF) grant with grant number NRF2016R1D1A1B03931090 (Y.K.), NRF-2017R1D1A1A09000951 (O.K.), NRF-2017R1D1A1B03032523 (D.T.), and NRF-2018R1D1A1 B07048061 (D.J.).

Open Access This article is distributed under the terms of the Creative Commons Attribution 4.0 International License (http://creativecomm ons.org/licenses/by/4.0/), which permits unrestricted use, distribution, and reproduction in any medium, provided you give appropriate credit to the original author(s) and the source, provide a link to the Creative Commons license, and indicate if changes were made.

Funded by SCOAP ${ }^{3}$.

\section{Appendix}

\section{A $C^{I_{1}=4}$ and $C^{(\Delta=2)}$}

In this Appendix, we determine the coefficients $C_{i_{1} \cdots i_{4}}^{I_{1}=4}$ which define the fourth scalar spherical harmonics on $S^{7}$ and the coefficients $C_{B_{1} B_{2}}^{A_{1} A_{2}}$ which defines the CPO of conformal dimension $\Delta=2$. To that end, we start from the definition of the fourth scalar spherical harmonics on $S^{7}$,

$Y^{4}=\frac{1}{L^{4}} \sum_{i, j, k, l=1}^{8} C_{i j k l} x^{i} x^{j} x^{k} x^{l}$

with the $\mathbb{R}^{8}$ coordinates $x^{i}$, s which are restricted to $S^{7}$ as follows,

$x^{1}=L\left(\frac{1+\tau}{2}\right)^{\frac{1}{2}} \cos \left(\frac{\theta}{2}\right) \cos \left(\frac{\phi+\psi}{2}\right)$,

$$
\begin{aligned}
& x^{2}=L\left(\frac{1+\tau}{2}\right)^{\frac{1}{2}} \cos \left(\frac{\theta}{2}\right) \sin \left(\frac{\phi+\psi}{2}\right), \\
& x^{3}=-L\left(\frac{1+\tau}{2}\right)^{\frac{1}{2}} \sin \left(\frac{\theta}{2}\right) \sin \left(\frac{\phi-\psi}{2}\right), \\
& x^{4}=L\left(\frac{1+\tau}{2}\right)^{\frac{1}{2}} \sin \left(\frac{\theta}{2}\right) \cos \left(\frac{\phi-\psi}{2}\right), \\
& x^{5}=L\left(\frac{1-\tau}{2}\right)^{\frac{1}{2}} \cos \left(\frac{\tilde{\theta}}{2}\right) \cos \left(\frac{\tilde{\phi}+\tilde{\psi}}{2}\right), \\
& x^{6}=L\left(\frac{1-\tau}{2}\right)^{\frac{1}{2}} \cos \left(\frac{\tilde{\theta}}{2}\right) \sin \left(\frac{\tilde{\phi}+\tilde{\psi}}{2}\right), \\
& x^{7}=-L\left(\frac{1-\tau}{2}\right)^{\frac{1}{2}} \sin \left(\frac{\tilde{\theta}}{2}\right) \sin \left(\frac{\tilde{\phi}-\tilde{\psi}}{2}\right), \\
& x^{8}=L\left(\frac{1-\tau}{2}\right)^{\frac{1}{2}} \sin \left(\frac{\tilde{\theta}}{2}\right) \cos \left(\frac{\tilde{\phi}-\tilde{\psi}}{2}\right) .
\end{aligned}
$$

The coefficients $C_{i j k l}$ are traceless under the contraction of any two indices and also are totally symmetric. Here we are interested in the scalar spherical harmonics on $S^{7}$ with $\mathrm{SO}(4) \times \mathrm{SO}(4)$ symmetry,

$Y^{4}=\tilde{\mathcal{N}}_{4}\left(1-5 \tau^{2}\right)$

where $\tilde{\mathcal{N}}_{4}$ is a normalization factor. Subsequently inserting (A.2) into (A.1), using the tracelessness and the symmetric conditions, and comparing with (A.3), we obtain

$$
\begin{aligned}
3 C_{1133} & =3 C_{1144}=C_{3333}=C_{1111}=4 \tilde{\mathcal{N}}_{4}, \\
3 C_{5577} & =3 C_{5588}=C_{7777}=C_{5555}=4 \tilde{\mathcal{N}}_{4}, \\
C_{1166} & =C_{1177}=C_{1188}=C_{3355}=C_{3366} \\
& =C_{3377}=C_{3388}=C_{1155}=-2 \tilde{\mathcal{N}}_{4},
\end{aligned}
$$$$
\text { the others }=0 \text {. }
$$

In order to determine the coefficients $C_{B_{1} B_{2}}^{A_{1} A_{2}}$ of the CPO of conformal dimension $\Delta=2$, we need to rewrite the scalar spherical harmonics in terms of $\mathbb{C}^{4}$ coordinates $y^{A}=$ $x^{2 A-1}+i x^{2 A}$ as

$Y^{4}=\frac{1}{L^{4}} \sum_{A, B, C, D=1}^{4} \widetilde{C}_{A B C D} y^{A} y_{B}^{\dagger} y^{C} y_{D}^{\dagger}$,

The coefficients $\widetilde{C}_{A B C D}$ satisfy the same conditions as $C_{i j k l}$ and the values of the former are determined from the values of the later as follows

$$
\begin{aligned}
& \widetilde{C}_{1111}=\frac{3}{8} C_{1111}+\frac{3}{4} C_{1122}+\frac{3}{8} C_{2222}=C_{1111}=4 \widetilde{\mathcal{N}}_{4}, \\
& \widetilde{C}_{2222}=\frac{3}{8} C_{3333}+\frac{3}{4} C_{3344}+\frac{3}{8} C_{4444}=C_{1111}=4 \widetilde{\mathcal{N}}_{4}, \\
& \widetilde{C}_{3333}=\frac{3}{8} C_{5555}+\frac{3}{4} C_{5566}+\frac{3}{8} C_{6666}=C_{1111}=4 \tilde{\mathcal{N}}_{4},
\end{aligned}
$$




$$
\begin{aligned}
& \widetilde{C}_{4444}=\frac{3}{8} C_{7777}+\frac{3}{4} C_{7788}+\frac{3}{8} C_{8888}=C_{1111}=4 \widetilde{\mathcal{N}}_{4}, \\
& \widetilde{C}_{1122}=\widetilde{C}_{1221}=\frac{3}{4}\left(C_{1133}+C_{1144}+C_{2233}+C_{2244}\right) \\
&=C_{1111}=4 \widetilde{\mathcal{N}}_{4}, \\
& \widetilde{C}_{3344}=\widetilde{C}_{3443}=\frac{3}{4}\left(C_{5577}+C_{5588}+C_{6677}+C_{6688}\right) \\
&=C_{1111}=4 \widetilde{\mathcal{N}}_{4}, \\
& \widetilde{C}_{1133}=\widetilde{C}_{1441}=\frac{3}{4}\left(C_{1155}+C_{1166}+C_{2255}+C_{2266}\right) \\
&=-\frac{3}{2} C_{1111}=-6 \widetilde{\mathcal{N}}_{4}, \\
& \widetilde{C}_{1144}=\widetilde{C}_{1441}=\frac{3}{4}\left(C_{1177}+C_{1188}+C_{2277}+C_{2288}\right) \\
&=-\frac{3}{2} C_{1111}=-6 \widetilde{\mathcal{N}}_{4}, \\
& \widetilde{C}_{2233}=\widetilde{C}_{2332}=\frac{3}{4}\left(C_{3355}+C_{3366}+C_{4455}+C_{4466}\right) \\
&=-\frac{3}{2} C_{1111}=-6 \widetilde{\mathcal{N}}_{4}, \\
& \widetilde{C}_{2244}=\widetilde{C}_{2442}=\frac{3}{4}\left(C_{3377}+C_{3388}+C_{4477}+C_{44488}\right) \\
&=-\frac{3}{2} C_{1111}=-6 \widetilde{\mathcal{N}}_{4}, \\
& \text { the others }=0 .
\end{aligned}
$$

Finally, we identify the coefficients $\widetilde{C}_{A B C D}$ with the coefficients $C_{B_{1} B_{2}}^{A_{1} A_{2}}$ of the $\mathrm{CPO}$ and thus can write

$$
\begin{aligned}
\mathcal{O}^{(\Delta=2)} & \sum_{A, B, C, D=1}^{4} \widetilde{C}_{A B C D} \operatorname{Tr}\left(Y^{A} Y^{\dagger B} Y^{C} Y_{D}^{\dagger}\right), \\
= & \mathcal{N}_{2}\left[\sum_{A, B=1}^{2} \operatorname{Tr}\left(Y^{A} Y_{A}^{\dagger} Y^{B} Y_{B}^{\dagger}\right)+\sum_{A, B=1}^{2} \operatorname{Tr}\left(Y^{A} Y_{B}^{\dagger} Y^{B} Y_{A}^{\dagger}\right)\right. \\
& +\sum_{A, B=3}^{4} \operatorname{Tr}\left(Y^{A} Y_{A}^{\dagger} Y^{B} Y_{B}^{\dagger}\right)+\sum_{A, B=3}^{4} \operatorname{Tr}\left(Y^{A} Y_{B}^{\dagger} Y^{B} Y_{A}^{\dagger}\right) \\
& -3 \sum_{A=1}^{2} \sum_{B=3}^{4} \operatorname{Tr}\left(Y^{A} Y_{A}^{\dagger} Y^{B} Y_{B}^{\dagger}\right) \\
& \left.-3 \sum_{A=1}^{2} \sum_{B=3}^{4} \operatorname{Tr}\left(Y^{A} Y_{B}^{\dagger} Y^{B} Y_{A}^{\dagger}\right)\right],
\end{aligned}
$$

where $\mathcal{N}_{2}=2 \tilde{\mathcal{N}}_{4}$

\section{References}

1. J. M. Maldacena, The large $\mathrm{N}$ limit of superconformal field theories and Int. J. Theor. Phys. 38, 1113 (1999) [Adv. Theor. Math. Phys. 2, 231 (1998)]. arXiv:hep-th/9711200

2. S.S. Gubser, I.R. Klebanov, A.M. Polyakov, Gauge theory correlators from noncritical string theory. Phys. Lett. B 428, 105 (1998). arXiv:hep-th/9802109

3. E. Witten, Anti-de Sitter space and holography. Adv. Theor. Math. Phys. 2, 253 (1998). arXiv:hep-th/9802150

4. K. Hosomichi, K.M. Lee, S. Lee, S. Lee, J. Park, N=5,6 superconformal Chern-Simons theories and M2-branes on orbifolds. JHEP 0809, 002 (2008). arXiv:0806.4977 [hep-th]

5. J. Gomis, D. Rodriguez-Gomez, M. Van Raamsdonk, H. Verlinde, A massive study of M2-brane proposals. JHEP 0809, 113 (2008). arXiv:0807.1074 [hep-th]

6. O. Aharony, O. Bergman, D.L. Jafferis, J. Maldacena, N=6 superconformal Chern-Simons-matter theories, M2-branes and their gravity duals. JHEP 0810, 091 (2008). arXiv:0806.1218 [hep-th]

7. H. Lin, O. Lunin, J.M. Maldacena, Bubbling AdS space and $1 / 2$ BPS geometries. JHEP 0410, 025 (2004). arXiv:hep-th/0409174

8. S. Cheon, H. C. Kim, S. Kim, Holography of mass-deformed M2branes. arXiv:1101.1101 [hep-th]

9. D. Jang, Y. Kim, O.K. Kwon, D.D. Tolla, Exact holography of the mass-deformed M2-brane theory. Eur. Phys. J. C 77(5), 342 (2017). arXiv: 1610.01490 [hep-th]

10. D. Jang, Y. Kim, O.K. Kwon, D.D. Tolla, Mass-deformed ABJM theory and LLM geometries: exact holography. JHEP 1704, 104 (2017). arXiv:1612.05066 [hep-th]

11. K. Skenderis, M. Taylor, Kaluza-Klein holography. JHEP 0605, 057 (2006). arXiv:hep-th/0603016

12. K. Skenderis, M. Taylor, Holographic Coulomb branch vevs. JHEP 0608, 001 (2006). arXiv:hep-th/0604169

13. K. Skenderis, M. Taylor, Anatomy of bubbling solutions. JHEP 0709, 019 (2007). arXiv:0706.0216 [hep-th]

14. S. Lee, S. Minwalla, M. Rangamani, N. Seiberg, Three point functions of chiral operators in $\mathrm{D}=4, \mathrm{~N}=4 \mathrm{SYM}$ at large $\mathrm{N}$. Adv. Theor. Math. Phys. 2, 697 (1998). arXiv:hep-th/9806074

15. G. Arutyunov, S. Frolov, Some cubic couplings in type IIB supergravity on $\operatorname{AdS}(5) \times S_{* * 5}$ and three point functions in SYM(4) at large N. Phys. Rev. D 61, 064009 (2000). arXiv:hep-th/9907085

16. D. Jang, Y. Kim, O. K. Kwon and D. D. Tolla, Gravity from Entanglement and RG Flow in a Top-down Approach. arXiv:1712.09101 [hep-th]

17. H.C. Kim, S. Kim, Supersymmetric vacua of mass-deformed M2brane theory. Nucl. Phys. B 839, 96 (2010). arXiv:1001.3153 [hepth]

18. R. Auzzi, S.P. Kumar, Non-Abelian vortices at weak and strong coupling in mass deformed ABJM theory. JHEP 0910, 071 (2009). arXiv:0906.2366 [hep-th]

19. K.K. Kim, O.K. Kwon, C. Park, H. Shin, Renormalized entanglement entropy flow in mass-deformed ABJM theory. Phys. Rev. D 90(4), 046006 (2014). arXiv:1404.1044 [hep-th]

20. K.K. Kim, O.K. Kwon, C. Park, H. Shin, Holographic entanglement entropy of mass-deformed Aharony-Bergman-Jafferis-Maldacena theory. Phys. Rev. D 90(12), 126003 (2014). arXiv:1407.6511 [hepth]

21. C. Kim, K.K. Kim, O.K. Kwon, Holographic entanglement entropy of anisotropic minimal surfaces in LLM geometries. Phys. Lett. B 759, 395 (2016). arXiv:1605.00849 [hep-th]

22. V. Balasubramanian, P. Kraus, A Stress tensor for Antide Sitter gravity. Commun. Math. Phys. 208, 413 (1999). arXiv:hep-th/9902121

23. S. de Haro, S.N. Solodukhin, K. Skenderis, Holographic reconstruction of space-time and renormalization in the AdS / 
CFT correspondence. Commun. Math. Phys. 217, 595 (2001). arXiv:hep-th/0002230

24. K. Skenderis, Asymptotically Anti-de Sitter space-times and their stress energy tensor. Int. J. Mod. Phys. A 16, 740 (2001). arXiv:hep-th/0010138

25. M. Bianchi, D.Z. Freedman, K. Skenderis, Holographic renormalization. Nucl. Phys. B 631, 159 (2002). arXiv:hep-th/0112119

26. M. Henningson, K. Skenderis, The holographic Weyl anomaly. JHEP 9807, 023 (1998). arXiv:hep-th/9806087

27. J. de Boer, E.P. Verlinde, H.L. Verlinde, On the holographic renormalization group. JHEP 0008, 003 (2000). arXiv:hep-th/9912012

28. P. Kraus, F. Larsen, R. Siebelink, The gravitational action in asymptotically AdS and flat space-times. Nucl. Phys. B 563, 259 (1999). arXiv:hep-th/9906127
29. M. Bianchi, D.Z. Freedman, K. Skenderis, How to go with an RG flow. JHEP 0108, 041 (2001). arXiv:hep-th/0105276

30. D. Martelli, W. Mueck, Holographic renormalization and Ward identities with the Hamilton-Jacobi method. Nucl. Phys. B 654, 248 (2003). arXiv:hep-th/0205061

31. K. Skenderis, Lecture notes on holographic renormalization. Class. Quant. Grav. 19, 5849 (2002). arXiv:hep-th/0209067

32. Y.H. Hyun, Y. Kim, O.K. Kwon, D.D. Tolla, Abelian projections of the mass-deformed ABJM theory and weakly curved dual geometry. Phys. Rev. D 87(8), 085011 (2013). arXiv:1301.0518 [hep-th] 